Tina Freyburg

\title{
Demokratisierung durch Zusammenarbeit?
}

Funktionale Kooperation mit autoritären Regimen und Sozialisation in demokratischem Regieren

Ist funktionale Kooperation mit autoritären Regimen ein Segen oder ein Fluch für Demokratisierung? Wissenschaftler vertreten überwiegend die Ansicht, dass Kooperation mit autoritären Regimen kontraproduktiv für Demokratisierungsprozesse sei, da sie das Regime stabilisiere. Dieser Artikel präsentiert empirische Befunde, die darauf schliessen lassen, dass funktionale Kooperation auch ein Weg sein kann, subtile Demokratisierungsprozesse auszulösen. Er untersucht, inwieweit Staatsbeamte in autoritären Regimen mit demokratischem Regieren vertraut werden, indem sie an transgouvernementalen Politiknetzwerken teilnehmen. Der empirische Fokus liegt dabei insbesondere auf dem Twinning-Programm, das von der Europäischen Union zur Implementierung funktionaler Kooperation mit der südlichen Nachbarschaft initiiert wurde. Die Studie führt Regressionsanalysen basierend auf originären Umfragedaten zur Einstellung marokkanischer Staatsbeamten zu demokratischem Regieren durch und ergänzt diese mit einem qualitativen Vergleich verschiedener Netzwerke. Die Ergebnisse stützen eine optimistische Einschätzung von funktionaler Kooperation. Indem sie die Einstellung zu demokratischem Regieren der involvierten Staatsbeamten signifikant beeinflusst, scheint Kooperation in der Tat einen demokratischen Wandel autoritärer Regime zu unterstützen.

\section{Einleitung}

Angesichts der Widerstandsfähigkeit ${ }^{1}$ stabiler autoritärer Regime warnte bereits ein neuer Forschungsstrang, die globale Welle der Demokratisierung könne nun verebbt

1 Eine frühere Version dieses Artikels wurde ausgezeichnet mit dem Preis der European Studies Association (EUSA) für das beste Konferenzpapier in 2009, dem Carl Beck Award 2010 der International Studies Association (ISA) sowie dem Preis der Standing Group International Relations des European Consortium for Political Research (ECPR) für das beste Konferenzpapier eines Doktoranden oder einer Doktorandin in 2010. Diese Studie profitierte von vielen hilfreichen Kommentaren. Ich danke insbesondere Stefanie Bailer, Tobias Böhmelt, Tanja Börzel, Sandra Lavenex, Janine Reinhard, Solveig Richter, Frank Schimmelfennig, Guido Schwellnus, Judith Vorrath, Rebecca Welge, Thomas Winzen, Jonas Wolff, den drei anonymen Gutachterinnen und Gutachtern sowie der ZIB-Redaktion sehr herzlich. Zu Dank verpflichtet bin ich ebenfalls der ETH European Politics-Gruppe, der Kolleg-Forschergruppe The Transformative Power of Europe und dem Forschungsnetzwerk »Externe Demokratisierungspolitik«. Ich möchte meinen Dank auch den zahlreichen europäischen und marokkanischen Beamten aussprechen, welche diese Studie ermöglicht haben sowie der FriedrichEbert-Stiftung in Rabat für ihre Gastfreundschaft und Assistenz. Diese Arbeit wurde finanziell durch den Schweizer Nationalfonds (SNSF) im Rahmen des National Centre for Competence in Research (NCCR) »Herausforderungen der Demokratie im 21. Jahrhundert» gefördert. 
sein. Wissenschaftler begannen folglich, vermehrt die Determinanten einer autoritären Konsolidierung in den Blick zu nehmen. ${ }^{2}$ Die Fähigkeit, autoritäre Herrschaft ohne Rückgriff auf Zwang aber durch einen gewissen Grad der Responsivität gegenüber innerstaatlichen Problemen aufrechtzuerhalten, wird von ihnen als Schlüssel für das Überleben nichtdemokratischer Regime angesehen. Autoritäre Herrscher seien interessiert, soziale und ökonomische Missstände zu bewältigen, da diese als Bedrohungen für die Stabilität des Regimes wahrgenommen werden. In der Hoffnung auf effektive Lösungen für gegenwärtige Herausforderungen öffnen sich diese Regime gegenüber funktionaler Kooperation mit wirtschaftlich und politisch liberalisierten Staaten. Problemspezifische Kooperation wird aus dieser Perspektive als kontraproduktiv für Demokratisierung angesehen: Indem sie dazu beiträgt, output-Legitimität zu generieren und die Stabilität des Regimes zu bewahren, hilft sie autoritären Regimen, an der Macht zu bleiben (vgl. Harders 2008: 39, 42; Schlumberger 2006: 38-39, 46-48; Albrecht/Schlumberger 2004: 376-377, 384-385).

Dieser Artikel nimmt eine andere Perspektive ein. Er betrachtet funktionale Kooperation als einen Ort der Sozialisation. Anstatt den Effekt zu untersuchen, den Kooperation auf Regimeebene erzielen könnte, strebt dieser Beitrag an, die black box der Mikroprozesse zu öffnen, die da stattfinden, wo Kooperation tatsächlich implementiert wird. Konkret werden transgouvernementale Netzwerke betrachtet, die funktionale Kooperation zwischen liberalen Demokratien und autoritären Regimen umsetzen. Ich untersuche den Effekt, den die Teilnahme an diesen Netzwerken auf die Einstellungen zu demokratischem Regieren von involvierten Staatsbeamten hat. Die Konzentration auf Sozialisation trennt Einstellungen von Verhalten. Sie ermöglicht damit, eine Aussage darüber zu treffen, ob funktionale Kooperation einen Einstellungswandel gegenüber demokratischem Regieren auslösen kann, auch wenn sich dieser (noch) nicht in effektivem Regimewandel zeigt. Die Ergebnisse zeigen, dass funktionale Kooperation in der Tat subtile Prozesse demokratischer Sozialisation hervorruft, die bisher außer Acht gelassen wurden. Im Lichte der anhaltenden Debatte in der internationalen Gemeinschaft über die Alternative einer Strategie der Isolation versus einer Strategie der Annäherung gegenüber nichtdemokratischen Staaten, fordern sie dazu auf, unser Wissen über die indirekten Effekte funktionaler Kooperation zu vertiefen.

Die demokratisierende Wirkung funktionaler Kooperation wird im Folgenden in einer empirischen Studie zu arabischen Staatsbeamten untersucht, die in transgouvernementale Netzwerke eingebunden sind/waren, die von der Europäischen Union (EU) im Rahmen ihrer Assoziierungspolitik gegenüber der südlichen Nachbarschaft initiiert und finanziert werden. Die Europäische Nachbarschaftspolitik (ENP) ist ein Paradebeispiel für eine Reformpolitik, die darauf abzielt, Interdependenzen zu gestalten und Integration auf der Ebene von Sektoren unterhalb der Mitgliedschaft zu stärken, indem sie vor allem danach strebt, die rechtlichen und administrativen Standards in den Nachbarstaaten denen der Union anzunähern (Freyburg et al. 2009:

2 Vgl. Burnell/Schlumberger (2010); Göbel/Lambach (2009); Brownlee (2007); Gandhi/Przeworski (2007); Leib/He (2006). 
916-917). Diese Regeln wurden für gefestigte Demokratien entwickelt und enthalten substantielle Elemente demokratischen Regierens. In dieser Hinsicht geht es bei Kooperation nicht nur darum, Problemlösungen zu erzielen und rechtliche Anpassungen vorzunehmen, sondern auch neue Regierungsmuster einzuführen. Die Umsetzung dieser funktionalen Kooperation findet in transgouvernementalen Netzwerken statt, die Spezialisten aus demokratischen und nichtdemokratischen Ländern zusammenbringen. Staatsbeamte, die in nichtdemokratischen Regierungsstrukturen angestellt sind, werden durch ihre Teilnahme an Aktivitäten dieser Netzwerke mit demokratischen Prinzipien der Entscheidungsfindung vertraut. Auf diese Weise kann Kooperation ein Potential für subtile Demokratisierungsprozesse freisetzen, die autonom von Demokratisierungsprozessen auf Regimeebene stattfinden. Langfristig jedoch könnte die Demokratisierung der Einstellungen zu angemessenen Entscheidungsfindungsprozessen in Teilen der Administration auf politische Institutionen der Regierungsebene übergehen. Wenn bürokratische und gesellschaftliche Akteure mit demokratischen Normen und Praktiken vertraut werden und beginnen, diese zunehmend in der administrativen Realität umzusetzen und einzufordern, könnte eine Dynamik entfacht werden, welche schließlich die Nachfrage nach weitreichender Demokratisierung des gesamten politischen Systems schafft.

Um demokratische Sozialisation durch funktionale Kooperation systematisch zu überprüfen, wende ich einen empirisch und methodisch innovativen Ansatz an. Empirisch bereichert diese Arbeit die Sozialisationsforschung durch eine Untersuchung des Arguments in einem bisher unerforschten Kontext. Methodisch investiert diese Studie darin, Einstellungen direkt zu messen anstatt sie aus Verhalten abzuleiten. Die bisherige Forschung konzentrierte sich weitgehend auf Prozesse, die entweder innerhalb internationaler oder regionaler Organisationen stattfinden ${ }^{3}$ oder durch diese Organisationen vor allem in Mittel- und Osteuropa nach der Implosion der Sowjetunion und dem Ende des Kalten Krieges ausgelöst wurden. ${ }^{4}$ Die vorliegende Studie führt funktionale Kooperation als einen Ort der Sozialisation transnationaler Normen ein. Sie nimmt hierfür transgouvernementale Netzwerke als Beispiel, die geschaffen wurden, um die Reformpolitik der EU gegenüber der südlichen Nachbarschaft umzusetzen. Die meisten Länder der Region des Mittleren Ostens und Nordafrikas stellen zum Zeitpunkt der Untersuchung eine in dieser Häufung einzigartige Kombination von Autoritarismus und starker Staatlichkeit dar, die eine erfolgreiche Demokratieförderung von außen äußerst unwahrscheinlich werden lassen. Es kann angenommen werden, dass die Einstellungen staatlicher Angestellter zu demokratischem Regieren eher negativ sind und dass potentielle Effekte demokratischer Sozialisation von innerstaatlichen Trends unterschieden werden können, da es unwahrscheinlich ist, dass diese Effekte in der Abwesenheit externer Einflüsse auftreten.

Methodisch geht diese Studie so vor, dass sie Einstellungen direkt erfasst anstatt sie aus Verhalten abzuleiten. Zu diesem Zweck entwickele ich eine originäre Skala, welche den Grad der individuellen Zustimmung zu demokratischen Normen des Re-

3 Kerr (1973); Checkel (2003); Beyers (2005); Hooghe (2005); Scully (2005).

4 Flockhart (2004); Gheciu (2005); Schimmelfennig et al. (2006). 
gierens misst, und untersuche einen neuen, selbst erhobenen Datensatz, der auf einer standardisierten Umfrage unter 150 marokkanischen Staatsbeamten basiert. Die Interpretation der Regressionsergebnisse wird durch Daten gestützt, die ich auf der Basis von 69 Interviews mit Vertretern der Regierung, von Nichtregierungsorganisationen, der Europäischen Kommission, von internationalen Organisationen und mit Journalisten und Wissenschaftlern in Marokko, Berlin, Wien und Brüssel zwischen 2007 und 2008 gewonnen habe.

Im ersten Teil des Artikels stelle ich den theoretischen Hintergrund dar und zeige den Zusammenhang zwischen funktionaler Kooperation und demokratischer Sozialisation auf. Im nachfolgenden Teil spezifiziere ich das methodische Vorgehen. Teil drei präsentiert die empirischen Befunde für das Argument und erforscht die Bedingungen, unter denen demokratische Sozialisation beobachtet werden kann. Die Ergebnisse unterstützen das Argument, dass funktionale Kooperation mit autoritären Regimen einen demokratisierenden Nebeneffekt auf die Einstellungen der beteiligten innerstaatlichen Akteure haben kann.

\section{Theorie: Demokratische Sozialisation und funktionale Kooperation}

Die Forschung zu internationaler Sozialisation kann entlang dreier Achsen klassifiziert werden: der Rolle (1) internationaler Institutionen in Sozialisationsprozessen, dem Gegenstand (2) und den Zielakteuren (3) von Sozialisation in/durch internationale Institutionen. Erstens, während einige Wissenschaftler Institutionen primär als Normförderer sehen, die versuchen, die Präferenzen und Einstellungen der Akteure mithilfe verschiedener Instrumente und Strategien zu beeinflussen (Risse et al. 1999; Finnemore 1993), nehmen andere eine strukturalistische Perspektive ein (Checkel 2005). Sie betrachten Institutionen als Orte der Sozialisation, in denen teilnehmende Akteure transnationale Normen infolge sozialer Interaktion und Kooperation internalisieren (Johnston 2001). Zweitens, Sozialisation selbst bezieht sich auf den Prozess, die zu Sozialisierenden in transnationale Normen wie Menschenrechte (Risse et al. 1999) und Demokratie (Flockhart 2004; Gheciu 2005) einzuführen, aber sie kann auch die Identität der Akteure betreffen. In letzterem Fall fragen Wissenschaftler primär, auf welche Weise die Mitgliedschaft in internationalen Organisationen dahingehend von Bedeutung ist, dass die Zugehörigkeit zu einer bestimmten Organisation Teil der Identitätsbildung wird (Hooghe 2005; Scully 2005; Kerr 1973). Drittens, Sozialisation kann sowohl auf der Makroebene der Staat(-sregierung) als auch auf der Mikroebene von Individuen wie Parlamentsabgeordneten oder nationalen Delegierten in internationalen Organisationen konzeptualisiert werden. 
Tabelle 1: Konzeptualisierungen von internationaler Sozialisation

\begin{tabular}{l|ll}
\hline & $\begin{array}{l}\text { Sozialisation durch inter- } \\
\text { nationale Institutionen }\end{array}$ & $\begin{array}{l}\text { Sozialisation in internationa- } \\
\text { len Institutionen }\end{array}$ \\
\hline $\begin{array}{l}\text { Rolle der internationalen Insti- } \\
\text { tutionen in Sozialisationspro- } \\
\text { zessen }\end{array}$ & Akteure/Förderer & Ort \\
$\begin{array}{l}\text { Gegenstand von Sozialisation } \\
\text { in/durch internationale Institu- } \\
\text { tionen }\end{array}$ & $\begin{array}{l}\text { Transnationale Normen, } \\
\text { z.B. Demokratie, Men- } \\
\text { schenrechte }\end{array}$ & $\begin{array}{l}\text { Internationale/Europäische } \\
\text { Identität }\end{array}$ \\
$\begin{array}{l}\text { Zielakteure von Sozialisation } \\
\text { in/durch internationale Institu- } \\
\text { tionen }\end{array}$ & Nationalstaaten & Individuen \\
\hline
\end{tabular}

Während die Studien, die Institutionen als Förderer betrachten, sich weitgehend auf Sozialisation von Staaten in transnationalen Normen konzentrieren, untersucht der Literaturstrang, der sich auf Institutionen als Orte der Sozialisation bezieht, vorwiegend Identitätswandel seitens der Individuen, die an internationale Organisationen delegiert werden. Dieser Artikel integriert beide Perspektiven, indem er zeigt, dass Individuen durch die Einbettung in internationale Institutionen auch in transnationalen Normen sozialisiert werden können (schattierte Fläche in Tabelle 1). Er betrachtet transgouvernementale Politiknetzwerke zwischen Verwaltungen liberaler Demokratien und autoritärer Regime als Ort der Sozialisation von Individuen in demokratischen Normen.

Die Anstrengungen, die in theoretisch und methodisch komplexe Studien der internationalen Sozialisation investiert wurden, haben (wenn überhaupt) nur moderate positive empirische Befunde hervorgebracht. ${ }^{5}$ Warum sollten wir dennoch demokratische Sozialisation bei »Härtefällen« wie Staatsbeamten in arabischen autoritären Regimen erwarten? Erstens impliziert Einstellungswandel zu demokratischem administrativen Regieren keinen Loyalitäts- oder Identitätswandel, der den Kern der Persönlichkeit eines Individuums berührt. Vielmehr betrifft er Normen, die Teil des professionellen Umfelds von Staatsbeamten sind. Zweitens werden demokratische Regierungsnormen als politisch sensitive Normen nicht direkt gefördert, sondern durch professionellen Austausch im Rahmen funktionaler Kooperation eingeführt. Drittens erlaubt eine Konzentration auf Einstellungswandel, unterschwellige externe Einflüsse zu erfassen, da funktionale Kooperation die Einstellung zu demokratischem Regieren von innerstaatlichen Akteuren formen mag, ohne - angesichts möglicher repressiver

5 Checkel (2005); Schimmelfennig (2003); Pollack (1998). Ausnahmen sind die positiven Befunde von Ghecius (2005) und Flockharts (2004) Studien zur Sozialisation von nationalen Vertretern der osteuropäischen Kandidatenstaaten in demokratischen Normen und Praktiken. Auch wenn die von der NATO geförderte Sozialisation im Schatten der Beitrittsperspektive erfolgte, deuten ihre Befunde in eine optimistischere Richtung. 
Konsequenzen - auch deren Realisierung in Verhalten auszulösen. Indem diese Studie solch subtile Prozesse individuellen Einstellungswandels untersucht, die bisher vernachlässigt wurden, ergänzt sie die existierende Forschung zu Sozialisation.

Das theoretische Argument verläuft wie folgt: Funktionale Kooperation zwischen der EU und ihren autoritären Nachbarn wird durch transgouvernementale Politiknetzwerke realisiert. Transgouvernementale Politiknetzwerke werden definiert als "pattern[s] of regular and purposive relations among like government units working across borders that divide countries from another and that demarcate the domestic from the international sphere « (Slaughter 2004: 14, Hervorh. dort). Gemäß dieser Perspektive bedeutet transgouvernemental, dass die Annahme, ein Nationalstaat sei ein einheitlicher Akteur, gelockert wird. Stattdessen legt sie nahe, die Bildung von Netzwerken zu betrachten, die auf der Ebene zwischen Regierung und Gesellschaft initiiert werden und zwischen Untereinheiten von Regierungen operieren, »when they act relatively autonomously from higher authority in international politics « (Keohane/ Nye 1974: 41). Diese Netzwerke konstituieren einen Ort der Sozialisation, indem sie Spezialisten aus den Verwaltungen der EU-Mitgliedstaaten und der Nachbarstaaten zusammenbringen. Das Ziel dieser Netzwerke ist, politikfeldspezifische Lösungen zu implementieren und die hierfür notwendigen rechtlichen Grundlagen in den ENPStaaten zu schaffen, wobei die rechtlichen und administrativen Standards der Union als Vorlage dienen. Da die zu transferierenden Regeln für etablierte Demokratien entwickelt wurden, beinhalten sie Elemente demokratischen Regierens (Freyburg et al. 2009: 916-917). Zudem vermitteln und gebrauchen die europäischen Experten, die in demokratischen Regierungsstrukturen professionell sozialisiert wurden, demokratisches Regieren, wenn sie als Experten im Ausland dienen. Als Teil ihres Beratungsdienstes sprechen sie auch Punkte an, die im innerstaatlichen Diskurs unterdrückt werden, wie die Teilnahme von nichtstaatlichen Akteuren an administrativen Entscheidungsfindungsprozessen und die Zugänglichkeit zu Informationen für die Öffentlichkeit. Auf diese Weise können ihre Amtskollegen mit Praktiken administrativen Regierens, wie sie in Demokratien ausgeübt werden, vertraut und in Prinzipien des demokratischen Regierens eingewiesen werden, die unter autoritärer Herrschaft kaum zu finden sind. Die Informationen, die in transgouvernementalen Netzwerken zur Verfügung gestellt werden, ermöglichen es ihnen, demokratische Formen des Regierens mit innerstaatlicher autoritärer Herrschaft zu vergleichen. Staatsbeamte könnten als Folge individuell und kollektiv danach streben, demokratische Formen des Regierens innerhalb staatlicher Verwaltungen durchzusetzen und langfristig Demokratisierungsprozesse auf Regimeebene voranzutreiben. Zusammengefasst könnte die funktionale Kooperation der EU mit nichtdemokratischen Nichtmitgliedstaaten »[be] capable of unleashing a dynamic of socialization around democratic norms" (Youngs 2001: 360). 


\subsection{Sozialisation in demokratischem Regieren}

Demokratische Sozialisation wird als ein Prozess des Einstellungswandels gegenüber demokratischem Regieren definiert, welcher hier als Folge des Kontaktes mit demokratischen Regeln und Praktiken administrativer Entscheidungsfindung entsteht. Sie liegt unter anderem dann vor, wenn Individuen ihre Einstellungen gegenüber demokratischen Normen aufgrund ihrer Erfahrungen in Politiknetzwerken ändern, ${ }^{6}$ die nicht in erster Linie darauf abzielen, Demokratie zu fördern. Der hier verwendete Begriff des demokratischen Regierens entspricht der Manifestation demokratischer Prinzipien in administrativen Alltagspraktiken. Er übernimmt den Gedanken, dass demokratische Prinzipien auf alle Situationen, in denen kollektiv bindende Entscheidungen beziehungsweise Entscheidungen für ein Kollektiv getroffen werden, anwendbar sind (Beetham 1999: 4-5; vgl. Dahl 1971: 12). Diese Prinzipien können folglich auf administrative Regeln und Praktiken auf der Ebene von Untereinheiten staatlicher Administration übertragen werden, selbst wenn diese in nichtdemokratische Staatsordnungen eingebunden sind. Im Unterschied zu gutem Regieren (good governance) (Kaufmann et al. 2005) geht es bei demokratischem Regieren (democratic governance) nicht darum, wie effektiv und effizient, sondern wie legitim die Regeln des politischen Spiels vereinbart und durchgesetzt werden (Hyden et al. 2004: 2; vgl. Coston 1998). Eine auf das Regieren ausgerichtete Demokratisierung erhöht die Chance, dass diejenigen, die von kollektiven administrativen Entscheidungen betroffen sind, die reelle Möglichkeit haben, diese Entscheidungen zu beeinflussen.

Um die Einstellungen staatlicher Beamter zu demokratischen Formen der Entscheidungsfindung zu messen, verwende ich ein multidimensionales Konzept von demokratischem Regieren. Dieses Konzept umfasst drei Dimensionen, entlang derer die Qualität demokratischen Regierens variieren kann: Transparenz, Verantwortlichkeit (accountability) und Partizipation (Freyburg et al. 2007). ${ }^{7}$ Transparenz bezieht sich auf die Bereitstellung und den Zugang zu verschiedenen Arten von Informationen für die allgemeine Öffentlichkeit (Zaharchenko/Goldenman 2004: 233). Verantwortlichkeit auf administrativer Ebene verweist auf die Pflicht von Beamten, ihr Handeln gegenüber den Bürgern und unabhängigen Drittparteien zu rechtfertigen sowie auf die Einrichtung und Anwendung von Verfahren administrativer Kontrolle inklusive Sanktionsmöglichkeiten im Falle einer Amtsverletzung (Grant/Keohane 2005: 29; Diamond et al. 1999: 3). Partizipation schließlich entspricht weitgehend dem Kernmerkmal des konventionellen Demokratieverständnisses auf der Ebene des Nationalstaates (Dahl 1971; Verba 1967). Übertragen auf administratives Regieren meint Partizipation, dass alle gewillten Teilnehmer des öffentlichen Lebens die gleiche und effektive Möglichkeit haben sollten, ihre Interessen und Bedürfnisse bekannt zu geben und auf diese Weise das Ergebnis der Entscheidungsfindung zu prägen. Obwohl

6 Diese Definition entspricht weitgehend dem klassischen Verständnis von Sozialisation als "process of inducting actors into the norms and rules of a given community (Checkel 2005: 804). Für eine Diskussion alternativer Definitionen siehe Pollack (1998) und Johnston (2001: 494-495).

7 Vgl. Bovens (2007); Hyden et al. (2004); Brinkerhoff (2000). 
die Übergänge zwischen diesen drei Dimensionen teilweise fließend sind, werden sie individuell analysiert. Zum einen ermöglicht dies, zu untersuchen, ob einige Dimensionen demokratischen Regierens zugänglicher für Sozialisation sind als andere; zum anderen setzt funktionale Kooperation in verschiedenen Sektoren jeweils auch den Schwerpunkt auf unterschiedliche Dimensionen. Während zum Beispiel das Einbeziehen betroffener Bürger und interessierter nichtstaatlicher Akteure insbesondere in Kooperationen zu Umweltangelegenheiten betont wird, wird das Einrichten von unabhängigen Kontrollautoritäten, die verantwortliches Regieren gewährleisten sollen, insbesondere in der Wettbewerbspolitik hervorgehoben.

\subsection{Arbeitshypothesen}

Die Idee, dass politikfeldorientierte Kooperation zwischen den öffentlichen Verwaltungen von liberalen Demokratien und autoritären Regimen möglicherweise Prozesse demokratischer Sozialisation hervorruft, basiert auf der Annahme, dass soziale Interaktion und Austausch auf gleicher Arbeitsebene von Bedeutung ist. Es wird daher erwartet, dass die Teilnahme an transgouvernementalen Politiknetzwerken einen positiven Einfluss auf die Einstellungen gegenüber demokratischem Regieren der Teilnehmer ausübt. In diesem Sinne lautet die Kernhypothese:

H1: Staatsbeamte, welche in transgouvernementale Politiknetzwerke involviert waren, habe eine positivere Einstellung zu demokratischem Regieren als ihre Kollegen, die nicht an diesen Netzwerken teilnahmen (»Kooperation«).

Die entsprechende Nullhypothese ist, dass die Teilnahme an transgouvernementalen Netzwerken keinen signifikanten Einfluss auf die Einstellungen zu demokratischem Regieren hat. Erstens, Wissenschaftler auf dem Gebiet der politischen Sozialisation betrachten Sozialisation in fundamentalen politischen Orientierungen gewöhnlich als einen Prozess, der vollständig durch innerstaatliche Bedingungen bestimmt ist. Ferner argumentieren sie, dass diese Orientierungen früh ausgebildet werden und im Laufe des Lebens relativ unverändert bleiben (Searing et al. 1976; Marsh 1971; Dawson/Prewitt 1969). Zweitens, gemäß rational choice-Theoretikern kann Kooperation nur die Rangordnung von Akteurspräferenzen ändern, nicht aber die Präferenzen und zugrundeliegenden Identitäten und Einstellungen selbst (Fearon/ Wendt 2003: 62-65; Moravcsik 1993).

Das demokratisierende Potential der Teilnahme an transgouvernementalen Netzwerken kann auch von anderen transnationalen Einflüssen abhängen. Studien zur Diffusion demokratischer Normen verweisen auf die besondere Rolle, die Austauschprogramme und ausländische Medien im Transfer von demokratischen Normen in nichtdemokratische Staaten spielen. Während Austauschprogramme ermöglichen, dass Bürger nichtdemokratischer Staaten Entscheidungsfindungsprozesse in demokratischen Staaten direkt erfahren können, ${ }^{8}$ transportieren ausländische Medien In-

8 Atkinson (2010); Pérez-Armendáriz/Crow (2010); Nye (2004). 
formationen über demokratische Praktiken über Grenzen hinweg. ${ }^{9}$ Staatsbeamte, die in nichtdemokratischen Staatsordnungen beschäftigt werden, können so bereits vor ihrer Teilnahme an transgouvernementalen Netzwerken Erfahrungen mit demokratischem Regieren gemacht haben. Studien zu internationaler Sozialisation identifizieren diesen »primacy effect « (Hooghe 2005: 866) als entscheidend für Sozialisation. Es wird erwartet, dass Staatsbeamte, die bereits Erfahrungen mit demokratischem Regieren gemacht haben, eher dazu geneigt sind, ihre Einstellungen zu ändern, wenn sie erneut in Kontakt mit demokratischem Regieren kommen (Johnston 2001: 497; Checkel 2001: 563). Auf Basis dieser Argumentation wird die Hypothese aufgestellt, dass eine sozialisierende Wirkung von funktionaler Kooperation wahrscheinlicher wird, wenn mindestens eine der genannten Bedingungen erfüllt ist.

Es ist wahrscheinlicher, dass die Teilnahme an transgouvernementalen Netzwerken einen positiven Einfluss auf die Einstellungen zu demokratischem Regieren von Staatsbeamten ausübt, wenn diese ...

$H 2 a \ldots$ längere Zeit in einer westlichen Demokratie zu Ausbildungs- oder beruflichen Zwecken verbracht haben (Auslandsaufenthalt);

$H 2 b \ldots$ regelmäßig westliche Medien zur politischen Information nutzen (ausländische Medien).

Die beiden transnationalen Einflüsse, Nutzung ausländischer Medien sowie Auslandsaufenthalt, werden sowohl als unabhängige Faktoren als auch als Interaktionsterme mit der Teilnahme an einem transgouvernementalen Netzwerk in der Analyse berücksichtigt.

\section{Forschungsdesign: Das Messen von Einstellungen zu demokratischem Regieren}

Diese Studie wendet ein sequenzielles, gemischtes Methodendesign an, welches quantitative Forschung durch eine qualitative Studie ergänzt (Morse 2002), um den demokratisierenden Effekt funktionaler Kooperation zu erfassen. Der erste Schritt ist quantitativ: Multiple Regressionsanalysen werden verwendet, um die Beziehung der Erklärungsvariablen, die sich auf die Eigenschaften der Staatsbeamten beziehen, zu den drei Dimensionen sowie dem übergeordneten Konzept des demokratischen Regierens zu erforschen. Ich führe somit multivariate Regressionsanalysen separat für Transparenz, Verantwortlichkeit, Partizipation und demokratisches Regieren insgesamt durch. Die Analysen beinhalten zuerst die Kernvariable dieser Studie - Teilnahme an einem Twinning-Projekt (»Kooperation«) als dichotome Variable, die misst, ob der individuelle Staatsbeamte in ein Projekt involviert war oder nicht. Anschließend wiederhole ich die Analysen, indem ich die Teilnahme an einzelnen ausgewählten Projekten als dichotome Variable einführe. Aufgrund seiner Robustheit gegenüber nicht-normalverteilten kontinuierlichen Daten verwende ich ein maximum-likelihood-Parameterschätzungsverfahren (MLMV), das Schätzungen mit Standardfehlern sowie Mittelwert- und Varianz-adjustierten Chi-Quadrat-Teststatistiken

9 Kern/Hainmueller (2009); Way/Levitsky (2007); Wejnert (2005); Whitehead (1996). 
liefert (Brown 2006: 379; Muthén/Muthén 2006: 426). ${ }^{10}$ In einem zweiten Schritt vergleiche ich systematisch die einzelnen Twinning-Projekte, um zu sondieren, welche Eigenschaften von transgouvernementalen Netzwerken demokratische Sozialisation unterstützen. Während multiple Regressionsanalysen der einzelnen TwinningProjekte ermöglichen, Unterschiede im Normentransfer zwischen den einzelnen Projekten aufzudecken, ermöglicht der nachfolgende qualitative Vergleich, spezifische Eigenschaften der unterschiedlichen Programme zur Erklärung empirischer Unterschiede heranzuziehen.

\subsection{Stichprobenauswahl und Datensammlung}

Die funktionale Kooperation der EU mit ihrer Nachbarschaft kann unterschiedliche Formen annehmen. Umweltkooperation in Marokko wurde beispielsweise lange durch regionale Programme implementiert, wie etwa das Umweltaktionsprogramm für Kurz- und Mittelfristige Prioritäten SMAP (Short and Medium-Term Priority Environmental Action Program) im Rahmen der Euro-mediterranen Partnerschaft, das transnationale LIFE-Drittstaatenprogramm, das durch das Sechste Umweltaktionsprogramm etabliert wurde, oder multilaterale Plattformen wie die mediterrane Komponente der EU-Wasserinitiative (EUWI). Mit der ENP wurden neue Instrumente für einen bilateralen administrativen Austausch eingeführt, vor allem das Programm für kurzzeitige technische Assistenz und Informationsaustausch TAIEX (Technical Assistance and Information Exchange Program), das zielgerichtete Unterstützung durch Experten anbietet. Zudem existiert das langzeitige Twinning-Programm, das als tool für Kooperation zwischen Untereinheiten der öffentlichen Verwaltung zu spezifischen Politikfragen fungiert.

Dieses Twinning-Programm eignet sich aufgrund der folgenden Eigenschaften besonders, den potentiell demokratisierenden Effekt funktionaler Kooperation zu untersuchen. ${ }^{11}$ Erstens, indem Twinning-Projekte Teil von Regieren in staatlichen Verwaltungen sind, gehören sie zur administrativen Realität. Ihr Ziel ist es, die begünstigten Abteilungen durch Training und Umstrukturierung sowie durch das Entwerfen von Gesetzen und Regulierungen nach dem EU acquis zu modernisieren. Die entwickelten Politiklösungen enthalten damit Elemente von demokratischem Regieren. Zweitens, im Gegensatz zu alternativen Politikreformprogrammen basieren Twinning-Projekte auf täglichen, intensiven Arbeitsbeziehungen über eine beträchtliche Zeitperiode. Dies hilft nicht nur, Beziehungen und damit Vertrauen und gegenseitiges Verständnis aufzubauen, sondern macht Staatsbeamte auch mit demokratischen administrativen Praktiken vertraut. Drittens, alle Projekte weisen den gleichen Grad struktureller Dichte auf und folgen der gleichen formalisierten Struktur, so dass ihre

10 Die MLMV-Schätzung basiert auf der MLM-Schätzung, die der Satorra-Bentler-Chi-Quadrat-Statistik entspricht.

11 Europäischer Rechnungshof (2003); Cooper/Johansen (2003: 6-7); Papadimitriou/Phinnemore (2003: 631). 
Vergleichbarkeit gewährleistet ist. Gleichzeitig sind die Projekte problemspezifisch und zeigen signifikante Unterschiede hinsichtlich ihrer Eigenschaften, wie etwa der Anzahl der begünstigten Abteilungen und dem Grad der Politisierung der betroffenen Politikfragen. Da es nicht mehr als ein Twinning-Projekt pro Untereinheit einer öffentlichen Verwaltung gibt, können überlappende Effekte mehrerer Twinning-Projekte ausgeschlossen werden; für die Effekte alternativer Kooperationsprogramme wird kontrolliert. Schließlich kann angenommen werden, dass mögliche Störeffekte durch selektive Rekrutierung marginal sind (Hooghe 2005; Pollack 1998; Kerr 1973). In den meisten Fällen war jeder Beamte, der in einer begünstigten Abteilung arbeitet, in mindestens eine der diversen Aktivitäten des Twinning-Projektes involviert. ${ }^{12}$ Die Teilnahme an den einzelnen Aktivitäten eines Twinning-Projektes wird auf der Grundlage objektiver Kriterien entschieden, wie etwa dem Zuständigkeitsbereich in der jeweiligen Abteilung und der professionellen Arbeitsleistung, nicht aufgrund von persönlichen Kontakten und Loyalitäten. ${ }^{13}$ Sollte ein Beamter daher ausnahmsweise nicht an Twinning-Aktivitäten teilgenommen haben, so lag dies in erster Linie daran, dass für seinen Aufgabenbereich (bisher) keine passende Aktivität angeboten wurde oder er aber an dem Termin verhindert war.

Der Effekt der Teilnahme an Twinning-Aktivitäten auf die Einstellung zu demokratischem Regieren wird am Beispiel Marokkos untersucht. Marokko gehört zu der Region des Mittleren Ostens und Nordafrikas, welche im weltweiten Vergleich, zumindest bis zum Zeitpunkt dieser Untersuchung, keine nennenswerten, rein endogenen Demokratisierungsprozesse erfuhr. Abbildung 1 gibt die Werte wieder, welche Freedom House für jedes Land in dieser Region in dem Zeitraum von 2000 bis 2008 vergibt. Die Abbildung zeigt deutlich, dass Marokko und Jordanien als autoritäre Regime bezeichnet werden können, auch wenn sie im regionalen Vergleich ein relativ hohes $\mathrm{Ma} ß$ an politischer Liberalisierung aufweisen. Jordanien und Marokko sind die einzigen Staaten, die Freedom House in dem genannten Zeitraum durchweg als »teilweise frei« klassifiziert. Im Zeitraum 2000 bis 2008 ergibt die zusammengefasste Bewertung der politischen Rechte und bürgerlichen Freiheiten auf einer Skala von 1 (»frei «) bis 7 (»nicht frei «) im Durchschnitt eine Bewertung von 4.7. Ein Minimum an Offenheit kann demnach erwartet werden (Al-Arkoubi/McCourt 2004: 983; Mohamedou 1999: 211), was die Wahrscheinlichkeit erhöht, dass administrative Kooperation demokratische Sozialisation hervorruft.

12 Nur wenige Beamte konnten folglich einen indirekten Sozialisationseffekt erfahren, indem sie sich mit ihren unmittelbaren Kollegen austauschten, die in einem Twinning-Projekt involviert waren.

13 Die Umfrage unter den Staatsbeamten beinhaltet die Frage: »Wie wichtig, denken sie, waren die folgenden Faktoren für ihre eigene Ernennung als Teilnehmer des TwinningProjektes?«. Die Antwortkategorien sind: »internationale Erfahrung«, »Sprachkenntnisse «, »Ausbildung «, »vorherige Zusammenarbeit mit der verantwortlichen Person«, »Persönlichkeit«, »professionelle Arbeitsleistung «, »persönliche Kontakte«, »Zuständigkeitsbereich in der Abteilung«, alle gemessen auf einer fünfstufigen Likert-Zustimmungsskala. Die gleiche Frage enthält auch eine Umfrage unter den europäischen Bürokraten, die in einem Twinning-Projekt in Marokko arbeiteten. Die Antworten wurden ergänzt durch Interviews mit Teilnehmern und Projektleitern/Experten der Twinning-Projekte. Deskriptive Statistiken werden gerne auf Anfrage zur Verfügung gestellt. 
Abbildung 1: Politische Rechte und bürgerliche Freiheiten in der arabischen Welt, 2000-2008

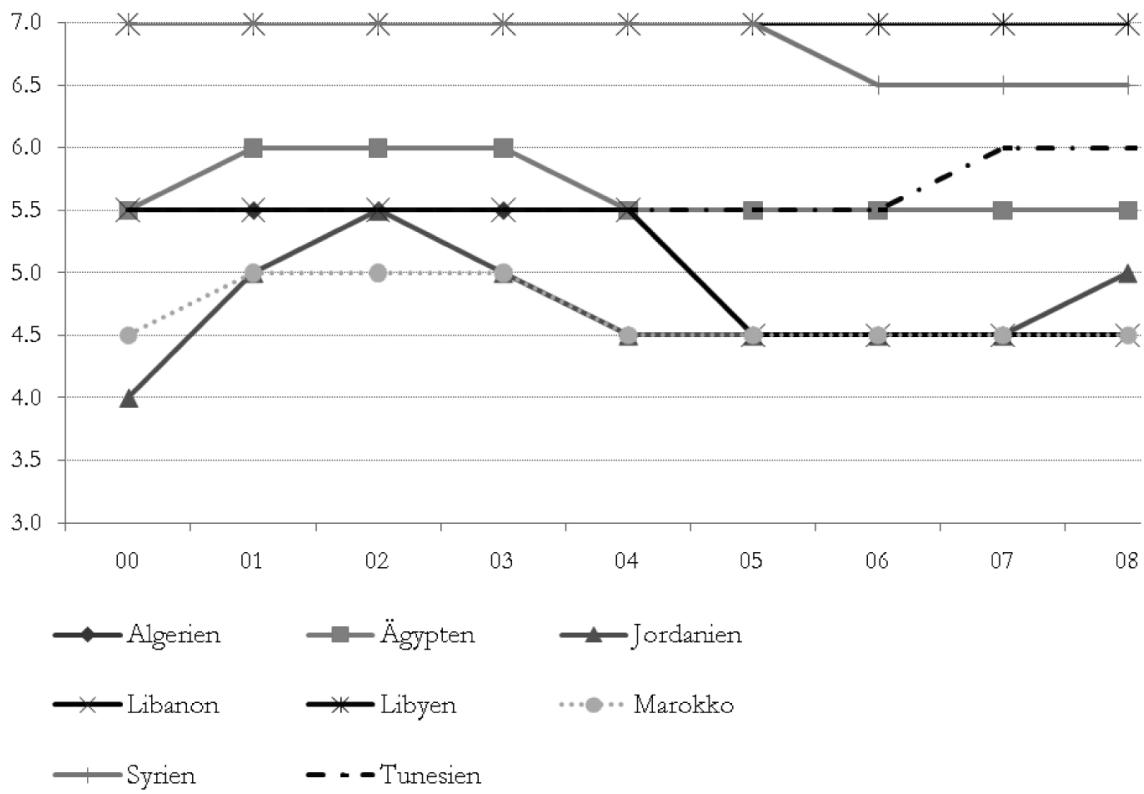

Deskriptive Statistiken; Freedom House klassifiziert Länder, deren durchschnittliche Bewertung des kombinierten Indexes von politischen Rechten und bürgerlichen Rechten zwischen 3.0 und 5.5 liegt als »teilweise frei« und zwischen 5.5 und 7.0 als »nicht frei«; der durchschnittliche Wert für Algerien liegt konstant bei 5.5; die Linie wird von den Linien für den Libanon (2000-04) und für Ägypten (2004-08) verdeckt.

Zudem ist das marokkanische Politiksystem als bürokratische Monarchie durch traditionelle, paternalistische Strukturen charakterisiert, welche staatlicher Bürokratie eine grosse Bedeutung für den Erhalt und die Stabilität des Regimes beimessen (Pawelka 2002; Zerhouni 2004). Marokko gehört außerdem zu den ersten Staaten der südlichen Nachbarschaft, die einen Aktionsplan der Europäischen Nachbarschaftspolitik (ENP) unterschrieben und Twinning-Projekte initiiert haben. Heute genießt das Land einen privilegierten Status (statut avancé) innerhalb der ENP. Wenn die Teilnahme in transgouvernementalen Netzwerken einen Einfluss auf die Einstellungen von Staatsbeamten in benachbarten autoritären Regimen ausübt, dann sollte ein solcher Effekt im Falle der EU-Twinning-Programme in Marokko auftreten. Im Falle eines negativen Befundes wiederum ist die Schlussfolgerung zulässig, dass administrative Kooperation, die weniger institutionalisiert ist und in Ländern stattfindet, die politisch weniger liberalisiert sind, keinen signifikanten Effekt zeigen wird.

Um die Einstellungen von marokkanischen Staatsbeamten zu demokratischem Regieren zu messen, erstellte ich einen Fragebogen mit dem Titel Administrative Regeln und Praktiken in öffentlichen Verwaltungen in Marokko. Die Befragten wurden durch 
ein theoretisch kontrolliertes Clusterverfahren ausgewählt: Alle Beamte, die in einer bestimmten Abteilung eines ausgewählten Ministeriums arbeiten, wurden gebeten, den Fragebogen auszufüllen. Die persönliche Verteilung des Fragebogens an die Beamten vor Ort ermöglichte eine Rücklaufquote von etwa 96\%. ${ }^{14}$ Eine Vollerhebung der selektierten Abteilungen war wichtig, um vorzubeugen, dass nur bestimmte, beispielsweise Europa-freundliche Beamte an der Befragung teilnahmen. Grundsätzlich wurden zwei Gruppen von Beamten gleichermassen berücksichtigt: Beamte, die an einem Twinning-Projekt teilnahmen $(N=85)$ und Beamte, die in einer thematisch verwandten Abteilung eines Ministeriums arbeiten, welches nicht von einem TwinningProjekt betroffen ist $(N=65) .{ }^{15}$ Die Differenz in der Einstellung zu demokratischem Regieren zwischen diesen beiden Gruppen wird dem Effekt der Teilnahme an Twinning-Projekten zugeschrieben, bei gleichzeitiger expliziter Kontrolle des möglichen Einflusses anderer relevanter Eigenschaften der Staatsbeamten (quasi-experimenteller statischer Gruppenvergleich, Campbell/Stanley 1966: 12). Das »fundamental problem of causal inference « (Holland 1986) ist, dass für jeden Befragten nie beide möglichen, sondern nur das tatsächliche Ereignis beobachtet werden kann. In anderen Worten, für jeden Befragten, der an einem EU-Twinning-Projekt teilnahm, kann nie das konterfaktische Niveau der Einstellung zu demokratischem Regieren beobachtet werden, welches er aufweisen würde, wenn er nicht teilgenommen hätte (und vice versa). Um einen unverzerrten Effekt zu erhalten, ist es entscheidend, eine geeignete Kontrollgruppe zu finden, welche der Fokusgruppe in allen relevanten Charakteristika genügend ähnelt, ausser, dass diese Gruppe nicht an Aktivitäten eines Twinning-Projektes teilgenommen hat.

\subsection{Die abhängigen Variablen}

Da diese Studie nicht auf bereits existierenden Umfragen aufbauen kann, bedurfte es der Entwicklung passender Indikatoren zu demokratischem Regieren, um die abhängigen Variablen messen zu können, das heisst die Einstellungen der Staatsbeamten zu demokratischem Regieren. Die drei theoretisch hergeleiteten Dimensionen demokratischen Regierens - Transparenz, Verantwortlichkeit und Partizipation - werden mittels Indikatoren operationalisiert, die auf verschiedene Aspekte administrativer

14 Aufgrund der Möglichkeit, unangenehme Fragen offen zu lassen, der garantierten Anonymität und dem nachdrücklichen Vorgehen blieben vollständige Verweigerungen fast vollkommen aus. Nur eine Beamtin weigerte sich, den Fragebogen auszufüllen; weniger als fünf Beamte konnten nicht erreicht werden, da sie berufliche Verpflichtungen im Ausland hatten oder im Urlaub waren. Es ist schwierig, für eine systematische Stichprobenverzerrung vollumfänglich zu testen, da soziodemographische Daten zu Staatsbeamten in Marokko nicht erhältlich sind. Die Befragten konnten die Kommunikationssprache selbst bestimmen (Französisch oder Arabisch), eine Geste, die sehr geschätzt wurde. Nur 9\% wählten jedoch die arabische Version.

15 Aufgrund der spezifischen und limitierten Natur der Zielgruppe wurde der Fragebogen durch Psychologen und politikwissenschaftliche Spezialisten auf dem Gebiet der arabischen autoritären Regime sowie Kollegen mit arabischem Migrationshintergrund kognitiv vorgetestet (Collins 2003; Presser et al. 2004). 
Partizipation

1 Ein Beamter sollte die Ansichten und Bedürfnisse der betroffenen Bürger vor dem Treffen einer Entscheidung berücksichtigen.

2 Ein Beamter sollte aktuelle Informationen zur Regierungspolitik zur Verfügung stellen.

3 Ein Beamter sollte sicherstellen, dass die Ansichten und Bedürfnisse der Bürger einen Einfluss auf die Formulierungen der Politiken haben.

n Ein Beamter sollte immer versuchen, die Öffentlichkeit in Einklang mit der Regierungspolitik zu bringen.

Transparenz

4 Ein Beamter sollte auf transparente und für die allgemeine Öffentlichkeit nachvollziehbare Weise arbeiten.

$5 \quad$ Ein Beamter sollte Bürgern die Möglichkeit bieten, ihre Ansichten als Beitrag zu Regierungsentscheiden vorzubringen.

6 Ein Beamter sollte Informationen für jeden zugänglich machen, der sie anfordert.

n Ein Beamter sollte sicherstellen, dass alle Informationen im Besitz öffentlicher Autoritäten allein in der Hand der Regierung verbleiben.

\section{Verantwortlichkeit}

$7 \quad$ Kontrolle durch unabhängige staatliche Institutionen sichert die Angemessenheit und prozedurale Korrektheit bürokratischer Entscheide.

8 Möglichkeiten für die allgemeine Öffentlichkeit und ihre Assoziationen, die Überprüfung der Entscheidungsfindungsprozesse und Politikentscheide einzufordern, sichert die Angemessenheit und prozedurale Korrektheit bürokratischer Entscheide.

n Anweisungen und Abnahme durch die höhere Autorität sichert die Angemessenheit und prozedurale Korrektheit bürokratischer Entscheide.

$n=$ negativ-orientierter Indikator

Entscheidungsfindung verweisen (siehe Tabelle 2). Konzeptionelle Arbeiten zu öffentlichen Verwaltungen und dem Zusammenhang zwischen (gutem) Regieren und wirtschaftlicher Entwicklung lagen den Formulierungen der einzelnen Indikatoren zugrunde. ${ }^{16}$ Alle Indikatoren wurden anhand einer fünfstufigen Likert-Zustimmungsskala gemessen. Um das Risiko von Antworttendenzen zu minimieren, wurden die Indikatoren zufällig in zwei der insgesamt 36 verschiedenen Fragensets des Fragebogens verteilt; ${ }^{17}$ einige der Indikatoren erscheinen umformuliert in verschiedenen

16 Vgl. Hyden et al. (2004); Baker (2002); Page (1985); Berger (1957).

17 Die zwei Fragensets werden folgendermassen eingeleitet: "Es gibt verschiedene Verständnisse darüber, was die Angemessenheit und prozedurale Korrektheit bürokratischer Entscheide in öffentlichen Verwaltungen bestimmt. Bis zu welchem Grad stimmen Sie persönlich zu, dass die folgenden Items dieser Funktion dienen? « (Indikatoren 7+8). »Es gibt verschiedene Ansichten darüber, was einen guten Beamten ausmacht. Bis zu welchem Grad stimmen Sie persönlich zu oder lehnen Sie persönlich ab, dass ein Beamter die folgenden Qualitäten aufweisen sollte?« (Indikatoren 1-6). 
Statements, andere sind Statements zu nichtdemokratischen Ausprägungen von Regieren (negativ orientierte Indikatoren).

Trotz dieses Fragebogendesigns und einem standardisierten Umfrageablauf können systematische Antwortverzerrungen nicht vollständig ausgeschlossen werden. Dies ist für diese Studie jedoch wenig problematisch, da ihr Interesse nicht primär darin liegt, das wahre Verständnis von angemessenem Regieren unter arabischen Staatsbeamten zu identifizieren. Vielmehr ist die Differenz in der Zustimmung zu demokratischem Regieren zwischen Staatsbeamten von Interesse, die an transgouvernementalen Netzwerken teilnahmen und solchen, die dies nicht taten. Im Allgemeinen kann nicht von einer systematischen Verzerrung der Antworttendenzen zwischen diesen beiden Gruppen ausgegangen werden; ein Sozialisationseffekt ist demnach nicht auf Effekte von Antworttendenzen zurückzuführen.

Mithilfe einer explorativen Faktorenanalyse (EFA) wurde die Passung der einzelnen Indikatoren zur Messung von Einstellungen innerhalb der drei Dimensionen demokratischen Regierens identifiziert. ${ }^{18}$ Eine EFA erlaubt, die Indikatoren zu bestimmen, welche für die Konstruktion von Skalen mit hoher Konsistenz geeignet sind, wie die interne Reliabilität jeder Skala bestätigt. ${ }^{19}$ Tabelle 2 zeigt den exakten Wortlaut der Indikatoren. Ich führte die EFA nur an den positiv-orientierten Indikatoren durch. Aufgrund einer Nichtbeantwortungsquote von etwa $10 \%$ zu den drei negativ orientierten Indikatoren ist die Anzahl fehlender Werte hier vergleichsweise hoch. Ihre Aufnahme hätte demzufolge die Anzahl der Fälle reduziert und zu einem erheblichen Verlust an Informationen zu den regulären Indikatoren geführt. Gemäß der theoretischen Annahme, dass ein »wahrer Demokrat« sowohl reguläre Items demokratischen Regierens unterstützt als auch ihre logischen Gegensätze ablehnt, werden

18 Für die explorative Faktorenanalyse wurden die oblique Rotationsmethode Oblimin und ein robustes Schätzverfahren verwendet, welches auf Mittelwert- und Varianz-adjustierten, gewichteten least squares (WLSMV) basiert. Die exakten Faktorenladungen sind in Anhang II gegeben.

19 Die Punktschätzung der Skalen-Reliabilität $(\rho)$ von Partizipation ist .79 (drei Indikatoren), von Verantwortlichkeit .58 (zwei Indikatoren) und von Transparenz .75 (drei Indikatoren), wenn Raykovs Methode angewandt wird, die auf einer konfirmatorischen Faktorenanalyse basiert. Dieser Ansatz ist nicht nur unempfindlich gegenüber Verletzungen der Annahme einer Normalverteilung, sondern liefert auch akkuratere Schätzungen der Reliabilität von Messungen mit mehreren Indikatoren als das übliche Kronbachs $\alpha$. Die Werte sind identisch interpretierbar (Sijtsma 2009; Raykov 2007; Brown 2006: 337-345). Kronbachs $\alpha$ von Partizipation ist .68, von Verantwortlichkeit .38 und von Transparenz .46. Aufgrund des explorativen Charakters dieser Studie, ihres Gegenstandes (Einstellungen und Präferenzen) und der geringen Anzahl von Indikatoren pro Skala, ist die Reliabilität der einzelnen Skalen auch dann akzeptabel, wenn der jeweilige theoretisch korrespondierende negativ orientierte Indikator jeder Skala hinzugefügt wird (vgl. John/Benet-Martinéz 2000: 346). Da diese Indikatoren nicht in die EFA eingeführt wurden, muss Kronbachs $\alpha$ anstelle des reliableren Ansatzes von Raykov verwendet werden. Kronbachs $\alpha$ ist .61 für Partizipation, .14 für Verantwortlichkeit und .30 für Transparenz. Die niedrigeren Werte entsprechen dem Rat, regulär formulierte und umgekehrt gepoolte Indikatoren nicht in einer einzigen Skala zu kombinieren, da dies die Reliabilität der Messung schmälern und somit die gewünschte Reduzierung von Antwortfehlern dämpfen könnte (vgl. Schriesheim et al. 1991; Pilotte/Gable 1990). Ich überprüfte diesen Effekt, indem ich die Regressionsanalysen anhand separater Skalen für positiv und negativ orientiere Indikatoren wiederholte; die Schätzergebnisse sind ähnlich und auf Anfrage erhältlich. 
die abhängigen Variablen anhand von Skalen gemessen, welche die positiv orientierten und negativ orientierten Indikatoren aggregieren. Jede Skala besteht demnach aus den positiv orientierten Indikatoren mit einer Faktorenladung von mindestens .40 in der Faktorenanalyse (Worthington/Whittaker 2006: 823) und dem jeweils theoretisch passenden negativ orientierten Indikator. Für jede Dimension demokratischen Regierens wurden Skalen konstruiert, indem die Werte der einzelnen Indikatoren addiert und die Summe durch die Anzahl der Indikatoren für jede Dimension geteilt wurde. Das übergeordnete Konzept des demokratischen Regierens wird anhand des Durchschnitts der drei individuellen Skalen gemessen.

Die Verteilung der abhängigen Variablen wird in Abbildung 2 gezeigt. ${ }^{20}$ Die dargestellten boxplots zeigen die Mittelwerte der abhängigen Variablen (50\% der erhobenen Daten), wobei die Endpunkte der vertikalen Linien (whiskers) den höchsten mit dem niedrigsten der Werte dieser Variablen verbinden. Die horizontalen Linien geben den Median an, die Kreuze das arithmetische Mittel. Die gestrichelte Linie zeigt das arithmetische Mittel von demokratischem Regieren als übergeordneter Kategorie (rechter Kasten). Da es einige wenige Ausreißer gibt, wie durch die Kreise gekennzeichnet, erstrecken sich die whiskers bis zu einem Maximum des 1.5-fachen des Interquartilsabstandes.

\section{Abbildung 2: Einstellung zu demokratischem Regieren}

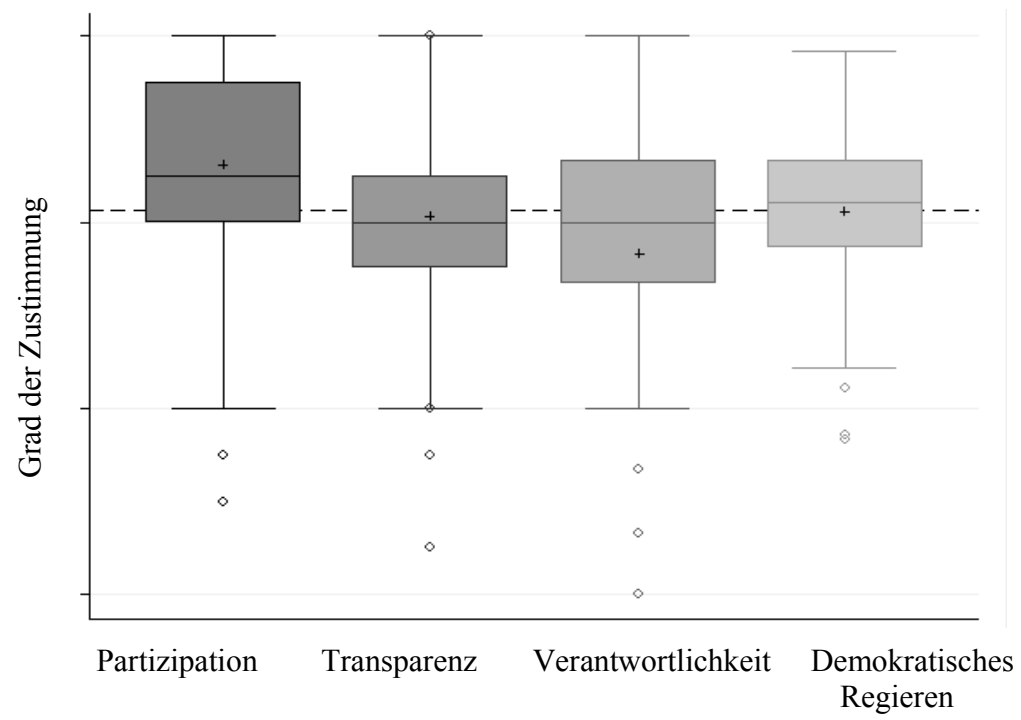

Boxplot; die Werte bewegen sich zwischen 1 (nichtdemokratisch) und 5 (demokratisch); $N=110$, Fälle mit fehlenden Werten wurden listenweise ausgeschlossen.

20 Für deskriptive Statistiken siehe Anhang Ia. 


\subsection{Variablen für die Regressionsanalysen (»Eigenschaften der zu Sozialisierenden«)}

Die unabhängige Kernvariable meiner Analyse ist die Teilnahme an einem Twinning-Projekt (»Kooperation«). Sie ist als eine binäre Variable kodiert, die den Wert 1 annimmt, wenn ein Beamter an einem Twinning-Projekt teilnahm, 0 wenn nicht. Das Modell wird durch die Einführung von zwei alternativen Erklärungsvariablen und zwei Kontrollvariablen vervollständigt, welche den individuellen Staatsbeamten charakterisieren.

Als alternative Erklärungsfaktoren führe ich zwei Variablen ein: »Auslandsaufenthalt in einer westlichen Demokratie« und die »Nutzung westlicher Medien«. Beide Variablen werden in neueren Studien häufig als Faktoren bezeichnet, die demokratische Normen in nichtdemokratische Staaten transferieren. In der vorliegenden Studie werden sie sowohl als unabhängige als auch als moderierende Variablen von Kooperation eingeführt. ${ }^{21}$ Die Nutzung von ausländischen Medien bezieht sich auf westliche Printmedien (Zeitungen und Magazine) sowie Fernsehkanäle. Die Befragten wurden gebeten anzugeben, welche Zeitungen/Magazine und Fernsehkanäle sie zur politischen Information ${ }^{22}$ in welcher Sprache nutzen und wie oft sie dies tun. Da ausländische Medienprodukte vor allem aus Europa kommen - etwa 97\% der gelesenen ausländischen Printmedien und 94\% der genutzten Fernsehkanäle - kann der erwartete Einfluss der Mediennutzung als europäisch geprägt bezeichnet werden. Mediennutzung wird als dichotome Variable operationalisiert, wobei 1 regelmäßige Mediennutzung repräsentiert.

Die Variable »Auslandsaufenthalt« verweist auf die internationalen Erfahrungen eines Beamten, operationalisiert als ein Aufenthalt von mindestens sechs Monaten in einem der »alten« Mitgliedstaaten der Europäischen Union und/oder in Nordamerika (NA) aus Ausbildungs- oder beruflichen Gründen. Diese Variable ist als dichotome Variable kodiert mit 1 für einen Aufenthalt in der EU und/oder in den Vereinten Staaten/Kanada. ${ }^{23}$ Da die Anzahl der Nordamerikabesucher sehr klein ist ( $N=9$ nur NA, $N=6$ NA und EU), werden Europa und Nordamerika in einer Kategorie zusammengefasst. Beamte, die eine bedeutende Zeit »im Westen « verbrachten, konsultieren westliche Medien nicht substantiell häufiger, oder umgekehrt. Die beiden Variablen

21 Die Regressionsanalyse ist ohne statistisch signifikante Unterschiede für alternative Kodierungen, z.B. nach Kategorien wie Fernsehen/Magazine getrennt, wiederholt worden. Es wurde jeweils die am wenigsten komplexe Lösung gewählt. Die Regressionsergebnisse sind auf Anfrage erhältlich. Anhang Ib zeigt die deskriptiven Statistiken und Interkorrelationen der unabhängigen Variablen.

22 Eine Studie zur gesellschaftlichen Unterstützung des ostdeutschen kommunistischen Regimes ergab sogar eine verstärkte Unterstützung des Regimes, wenn ausländische Medien primär zur Unterhaltung genutzt wurden (Kern/Hainmueller 2009).

23 Der nichtparametrische Kruskal-Wallis-Test, der einen Vergleich der Rangwerte der Mittelwerte für die drei Dimensionen ermöglicht, ergab keine signifikanten Unterschiede in der Einstellung zu demokratischem Regieren von Beamten, die ihren Aufenthalt in Europa und solchen, die ihn in Nordamerika oder beiden Zielregionen verbrachten $\left(\mathrm{df}=2 ; x^{2}=.310\right.$, $\mathrm{p}=.856$ für Partizipation; $x^{2}=1.913, \mathrm{p}=.384$ für Verantwortlichkeit; $x^{2}=.208, \mathrm{p}=.901$ für Transparenz). 
stehen folglich nicht in einer signifikanten Wechselbeziehung (siehe Korrelationsmatrix in Anhang Ib).

Zusätzlich zu den drei unabhängigen Variablen enthält das Modell zwei Kontrollvariablen: »administrative Sozialisation« und vorherige Teilnahme an »alternativen Programmen«. Es wird erwartet, dass Beamte, die in die öffentliche Verwaltung eintraten, nachdem reformorientiertere Kräfte die Regierung übernommen haben und/ oder die bereits Wissen über demokratisches Regieren durch die Teilnahme in vorherigen Politikreformprogrammen erworben haben, die von westlichen Gebern initiiert und durchgeführt wurden, wahrscheinlich eine positivere Einstellung zu demokratischem Regieren aufweisen. Diese Vermutung trägt einer Vorsozialisation Rechnung (vgl. Beyers 2005; Checkel 2005: 813; Johnston 2005; Hooghe 2005). Insbesondere höhere Beamte als »/well-connected members of the old guard « (Baker 2002: 293, Hervorh. dort) können demokratisches Regieren als tatsächliche Bedrohung ihrer Privilegien wahrnehmen. Demgegenüber sind Beamte, die erst kürzlich in die jeweilige administrative Untereinheit eingestellt wurden, weniger in die vorherrschende Herrschaftskultur eingebunden und daher eher geneigt, sich nach demokratischen Formen des Regierens zu richten (Checkel 2001: 562; Johnston 2001: 497; Flockhart 2004). Administrative Sozialisation wird durch die Anzahl der Berufsjahre unter dem »neuen« König Mohammed VI operationalisiert, das heisst mehr Dienstjahre unter dem gegenwärtigen als unter dem vorherigen Regime (0) beziehungsweise mehr Dienstjahre unter dem vorherigen Regime von König Hassan II (1). Grund für diese Kodierung ist, dass das politische Regime unter König Hassan II während seiner langen Regierungszeit (1961-99) durch »control over both the technocratic state apparatus and the army and the police« (Desrues/Moyano 2001: 21) gekennzeichnet war. Mit der Thronbesteigung von Mohammed VI in 1999 zog ein neuer Geist der politischen, sozialen und wirtschaftlichen Reformen in das Land ein, während gleichzeitig das tatsächliche Potential eines bedeutungsvollen demokratischen Wandels begrenzt blieb (Zerhouni 2004; Campbell 2003). Die Teilnahme an vorherigen Politikreformprogrammen wird als binäre Variable behandelt mit dem Wert 1, wenn der Beamte an mindestens einem Programm teilgenommen hat. 46\% der Befragten nahmen an einem Programm teil, das von einer Entwicklungsagentur eines der EU-Mitgliedstaaten eingerichtet wurde, insbesondere von der Französischen Entwicklungsagentur (AFD) und der Deutschen Gesellschaft für Technische Zusammenarbeit (GTZ). 36\% waren an Aktivitäten der Weltbank beteiligt, 23.2\% an Projekten der USamerikanischen Agentur für Internationale Entwicklung (USAID), 20\% an denen der Japanischen Internationalen Entwicklungsagentur (JICA) und 18.5\% am Entwicklungsprogramm der Vereinten Nationen (UNDP). Die vorherige Teilnahme an anderen Programmen als dem EU-Twinning-Programm stellt einen Faktor dar, der unabhängig von »Kooperation« ist (siehe Korrelationsmatrix in Anhang Ib). Auch hier wurden die Variablen als konstitutive und Interaktionstermini eingeführt. 


\subsection{Merkmale für die vergleichende Analyse (»Eigenschaften der Politiknetzwerke als Orte der Sozialisation")}

Es wird erwartet, dass nicht nur die Eigenschaften der individuellen Staatsbeamten, sondern auch die Eigenschaften der individuellen transgouvernementalen Netzwerke die Wahrscheinlichkeit von Sozialisation in demokratischem Regieren bestimmen. Wenn wenige Abteilungen eingebunden werden (»Größe«), externe Experten für eine lange Zeitdauer bleiben (»Dauer «) und Interaktion in »less politicized and more insulated, in-camera settings « (Checkel 2003: 213) stattfindet, dann ist die Interaktion zwischen den Teilnehmern intensiver und vertrauenswürdiger. ${ }^{24}$ Es kann daher erwartet werden, dass ein Einstellungswandel zu demokratischem Regieren wahrscheinlicher wird. Anhand dieser drei Eigenschaften sollen die Unterschiede des Sozialisationseffektes zwischen den einzelnen Twinning-Projekten in Marokko erklärt werden.

Anstatt Werte für jeden einzelnen Staatsbeamten zu bestimmen, werden die drei Variablen mithilfe indirekter Maße auf der Ebene der Netzwerke operationalisiert. Die Größe des Netzwerkes wird als die Anzahl der begünstigten Abteilungen und Ministerien operationalisiert. Die Dauer bezieht sich auf die Länge des TwinningProjektes in Monaten zum Zeitpunkt der Umfrage. Die Projekte werden hinsichtlich Netzwerkgröße und -dauer klassifiziert, indem die höchste Anzahl involvierter Einheiten und die längste Periode als »hoch « und das kleinste Netzwerk und die kürzeste Interaktion entsprechend als »niedrig « gekennzeichnet werden. Netzwerke, die zwischen diese beiden Kategorien fallen, werden als »mittel« klassifiziert. Schließlich geht es bei Politisierung um die Bedeutung einer Politikfrage für die Integrität des Staates und die Aufrechterhaltung politischer Macht seitens der herrschenden Elite. Obwohl transgouvernementale Politiknetzwerke gemeinhin ohne viel Öffentlichkeit operieren und von Turbulenzen politischer Auseinandersetzungen relativ unberührt sind (Pollack 2005: 906; Slaughter 2000: 200-202), ist funktionale Kooperation dennoch eingebunden in politische Prozesse und von politischen Interessen abhängig. Interviews mit marokkanischen Staatsbeamten, Journalisten und nichtstaatlichen Aktivisten sowie Vertretern internationaler Organisationen, EU-Mitgliedstaaten und der Delegation der Europäischen Kommission halfen, den Grad der Politisierung in den untersuchten Politikbereichen als »hoch«, »mittel« oder »niedrig« einzustufen. Indikatoren hierfür sind, inwiefern die Medienberichterstattung pluralisiert ist und sektorale Kooperation von machtpolitischen Erwägungen beeinträchtigt wird. Wettbewerbspolitik kann beispielsweise als politisiert angesehen werden, da es sensitive Bereiche wie Korruption, Klientelismus und die Verknüpfung von Privatwirtschaft und Regierungsverantwortlichkeiten berührt. Diese drei Eigenschaften der Politiknetzwerke - Größe, Dauer und Grad der Politisierung - werden im Anschluss an die folgende Regressionsanalyse zur Erklärung der unterschiedlichen Sozialisationseffekte in den einzelnen Twinning-Projekten verwendet.

24 Vgl. Slaughter (2004: 198-200); Checkel (2003: 210); van Waarden (1992); Marsden (1990). 


\section{Empirie: Funktionale EU-Kooperation und demokratische Sozialisation in Marokko}

Um den Effekt der Teilnahme an einem Twinning-Projekt auf die Einstellungen der teilnehmenden Beamten zu schätzen, rechnete ich vier Modelle. In Modell 1 werden die abhängigen Variablen, das heißt die drei individuellen Dimensionen demokratischen Regierens und das übergeordnete Konzept, jeweils auf die Twinning-Variable regressiert; Modell 2 kontrolliert zusätzlich für den Effekt der vier Erklärungsvariablen, die sich auf die Eigenschaften der Staatsbeamten beziehen. In den Modellen 1 und 2 (Hypothese 1) wurden Interaktionsbedingungen nicht berücksichtigt. Um die bedingten Effekte zu testen (Hypothesen 2 a und 2 b), nehme ich die Kreuzprodukte der Twinning-Variable und von jedem einzelnen Erklärungsfaktor als binäre Variable in die Analyse auf (Brambor et al. 2006: 73; Braumoeller 2004). Tabelle 3 zeigt nur die signifikanten Interaktionseffekte in den Modellen 3 a und 3 b. Im Kontrollmodell werden die Variablen des demokratischen Regierens allein auf die Eigenschaften der Staatsbeamten regressiert (Modell 4).

\subsection{Der sektorenübergreifende demokratisierende Effekt von funktionaler EU- Kooperation}

Tabelle 3 präsentiert die Schätzergebnisse für die Wahrscheinlichkeit, dass ein Twinning-Projekt die Einstellungen der Teilnehmer zu demokratischem Regieren beeinflusst. Die Ergebnisse stützen das Argument vom demokratisierenden Potential funktionaler Kooperation. Sie zeigen jedoch auch auf, dass allein die Teilnahme an einem Twinning-Projekt die Einstellungen der involvierten Beamten nicht signifikant prägt (Modell 1). Der Koeffizient für Kooperation ist zwar positiv, aber nicht signifikant. ${ }^{25}$ Hypothese 1, welche den unabhängigen Effekt funktionaler Kooperation postuliert, muss damit zurückgewiesen werden. Interessanterweise hat die Teilnahme an Politikreformprogrammen im Allgemeinen jedoch einen signifikanten unabhängigen Effekt auf die Einstellung zu demokratischem Regieren. Der Koeffizient für »andere Programme« in Modell 2 ist positiv und signifikant. Diese positive Korrelation ist jedoch nicht notwendigerweise das Ergebnis der Teilnahme, sondern kann auch durch die Häufung dieser Programme in weniger politisierten Bereichen hervorgerufen worden sein. 58.4\% der Projekte der drei wichtigsten externen Akteure aus der westlich-demokratischen Hemisphäre neben der EU und ihren Mitgliedstaaten - die Vereinigten Staaten von Amerika (USAID), Weltbank und Japan, an denen die befragten

25 Dieser Befund unterstützt die Beobachtung, dass die Teilnahme an einem Twinning-Projekt nicht durch bestimmte Eigenschaften wie eine besonders positive oder negative Einstellung der Beamten gegenüber der EU oder demokratischen Prinzipien begründet und dadurch womöglich ein konfundierender Effekt für eine demokratische Sozialisation in TwinningProjekten ist. 
Staatsbeamten teilgenommen haben, fanden in Politikfeldern wie Umwelt, Gesundheit und Bildung statt. ${ }^{26}$

Tabelle 3: Ergebnisse der Regressionsanalyse - Demokratisches Regieren

\begin{tabular}{|c|c|c|c|c|c|}
\hline & (1) & (2) & (3a) & (3 b) & (4) \\
\hline Kooperation & $.084(.074)$ & $.068(.075)$ & $-.092(.095)$ & $.272(.114)^{*}$ & \\
\hline Admin. Sozialisation & & $-.036(.073)$ & $-.042(.072)$ & $-.042(.072)$ & $-.045(.074)$ \\
\hline Auslandsaufenthalt & & $-.052(.075)$ & $-.251(.119)^{*}$ & $.251(.119)^{*}$ & $-.049(.075)$ \\
\hline Ausländ. Medien & & $-.085(.092)$ & $-.055(.090)$ & $-.055(.090)$ & $-.062(.089)$ \\
\hline Andere Programme & & $.217(.077)^{* * *}$ & $.218(.074)^{* * *}$ & $.218(.074)^{* * *}$ & $.221(.077)^{* * *}$ \\
\hline $\begin{array}{l}\text { Koop x 1: Auslands- } \\
\text { aufenthalt }\end{array}$ & & & $.364(.148)^{*}$ & & \\
\hline $\begin{array}{l}\text { Koop x 1: kein Auslands- } \\
\text { aufenthalt }\end{array}$ & & & & $-.364(.148)^{*}$ & \\
\hline$R^{2}$ & .012 & .096 & .152 & .152 & .089 \\
\hline$A I C$ & 236.286 & 764.886 & 762.090 & 762.090 & 623.859 \\
\hline Log Likelihood & -128.643 & -375.443 & -373.045 & -373.045 & -305.929 \\
\hline$N$ & 110 & 103 & 103 & 103 & 103 \\
\hline
\end{tabular}

Multiple Regressionsanalyse (MLMV); Regressionskoeffizienten sind nicht standardisiert; Standardfehler in Klammern; Fälle mit fehlenden Werten listenweise ausgeschlossen; ${ }^{*} p \leq .05$, ** $\leq .01$.

Entgegen der gängigen Meinung, dass die Nutzung westlicher Medien und Auslandsaufenthalte einen demokratisierenden Effekt haben, weisen meine Ergebnisse darauf hin, dass Staatsbeamte, die diesen transnationalen Einflüssen ausgesetzt waren, demokratischem Regieren gegenüber nicht aufgeschlossener sind. Vielmehr ist in beiden Fällen der Koeffizient sogar (wenn auch nicht signifikant) negativ (Modell 2). Während für die Nutzung ausländischer Medien kein signifikanter bedingter Effekt von Twinning-Aktivitäten beobachtet werden kann, ist der Koeffizient des Interaktionsterms von Kooperation und Auslandsaufenthalt in Modell 3 a positiv und statistisch signifikant, wie auch ein Wald-Test bestätigt ( $p=.000 ; \mathrm{df}=3 ; x^{2}=4793.418$ ). Dieser Befund stützt Hypothese $2 \mathrm{a}$, aber nicht Hypothese $2 \mathrm{~b}$. Um das Verständnis der Staatsbeamten von angemessenem Regieren signifikant beeinflussen zu können, scheint es demnach, als ob die Aktivitäten der Twinning-Programme Staatsbeamten zu Gute kommen müssen, die bereits demokratische Verfahren in westlichen Demokratien persönlich erlebt haben. Da die Beamten während ihres Studiums im Ausland waren, das heißt bevor sie in die staatliche Verwaltung eintraten, ist der marginale Effekt der Teilnahme an einem Twinning-Projekt auf die Einstellung von Beamten mit internationaler Erfahrung positiv (siehe Koeffizienten in Model 3: -.092+.364=. 272; vgl. Brambor et al. 2006: 73). Für den Einfluss von transgouvernementalen

26 United States Agency for International Development (2011); Organisation für Wirtschaftliche Zusammenarbeit und Entwicklung (2011). 
Netzwerken auf die Einstellung zu demokratischem Regieren von Staatsbeamten in autoritären Regimen - und dieser Effekt steht im Fokus dieses Papieres - bedeutet dieser Befund vor allem, dass ein vorheriger direkter Kontakt mit demokratischen Prinzipien - unabhängig von ihrer Bewertung - notwendig zu sein scheint, damit ein späterer zielgerichteter Austausch über diese Prinzipien an bereits vorhandene Schemata, das heisst mentale Wissensstrukturen anknüpfen, diese aktivieren und deuten kann.

Die Ergebnisse der Regression zu den einzelnen Dimensionen spiegeln die Befunde der Regression zu dem übergeordneten Konzept des demokratischen Regierens wider (siehe Anhang III). Während die Regression zu partizipatorischem Regieren ein ähnliches Muster signifikanter Regressionskoeffizienten ergibt, unterscheiden sich die Ergebnisse $\mathrm{zu}$ verantwortlichem und transparentem Regieren dahingehend, dass Twinning-Programme weder einen unabhängigen noch einen bedingten Effekt hervorrufen.

\subsection{Der demokratisierende Effekt individueller Twinning-Projekte}

Zum Zeitpunkt der Untersuchung gab es in Marokko neun Twinning-Projekte, die bereits mindestens ein Jahr liefen, ${ }^{27}$ aus denen die folgenden vier für die empirische Analyse ausgewählt wurden: Das Twinning-Projekt Koordiniertes Umweltmanagement und die Harmonisierung der nationalen Umweltgesetzgebung (MA04/AA/ EN03), das Projekt Entwicklung und Durchführung legislativer, organisatorischer und technologischer Maßnahmen zur Sicherung eines freien Handelsaustausches an den Grenzen (MA04/AA/FI01), das Projekt Unterstützung der Stärkung der Wettbewerbsbehörden (MA06/AA/FI08) und das Projekt Stärkung der Organisationen für veterinäre und phytosanitäre Gesundheitskontrollen (MA06/AA/HE06). Diese vier Twinning-Projekte unterscheiden sich hinsichtlich der identifizierten Eigenschaften, das heißt in ihrer Größe, Dauer und dem Grad der Politisierung des Politikbereiches.

Das Twinning-Projekt im Bereich Umwelt weist die günstigsten Bedingungen auf: einen niedrigen Grad an Politisierung und ein mittelgroßes Netzwerk. Das Projekt Entwicklung und Durchführung legislativer, organisatorischer und technologischer Maßnahmen zur Sicherung eines freien Handelsaustausches an den Grenzen hingegen, das insbesondere die marokkanische Behörde für Zölle und indirekte Steuern betrifft, sieht sich mit einem hohen Grad an Politisierung und einer großen Anzahl begünstigter Abteilungen konfrontiert. Zwischen diesen beiden Projekten rangieren das Wettbewerbsprojekt und das Projekt zu Gesundheitskontrollen. Eine Übersicht zu den Eigenschaften gibt Tabelle 4. Die Politikbereiche Wettbewerb und Zölle können als hochgradig politisiert behandelt werden, da sie sensitive Fragen wie Korruption, Klientelismus und die Verknüpfung von privaten wirtschaftlichen Geschäften und Regierungsverantwortlichkeiten berühren. In Politikfeldern, die öffentliche Güter bereitstellen wie Umwelt und zu einem geringen Maße Gesundheitskontrolle, ist die

27 Eine Liste der Projekte ist auf Anfrage erhältlich. 
Medienberichterstattung pluralisierter und transgouvernementale Kooperation weniger durch politische Erwägungen beeinträchtigt, beides Indikatoren für einen vergleichsweise geringen Grad an Politisierung.

Regressionsanalysen für die einzelnen Projekte beleuchten das demokratisierende Potential des Twinning-Programmes im Detail. Auch wenn diese Analysen insgesamt denselben Effekt der Teilnahme an einem Twinning-Projekt in Abhängigkeit eines Auslandsaufenthaltes wie die obige allgemeine Regressionsanalyse zeigen, so werden gleichzeitig die Unterschiede zwischen den einzelnen Projekte deutlich (siehe Anhang IV). Während das Umweltprojekt einen signifikanten unabhängigen Effekt auf die Einstellungen zu demokratischem Regieren der teilnehmenden Beamten hat, beeinflussen die Projekte zu Wettbewerbspolitik und zu Gesundheitskontrolle diese Einstellungen nur dann signifikant, wenn die teilnehmenden Beamten bereits im demokratischen Ausland waren. Das Zollprojekt hingegen hat weder einen signifikanten unabhängigen noch bedingten Effekt. Die Analyse der einzelnen Projekte bringt darüber hinaus zwei weitere Ergebnisse hervor. Erstens, der Effekt der Teilnahme an einem Twinning-Projekt auf die Einstellungen zu demokratischem Regieren ist sektorspezifisch. Die Einstellungen zu den Subdimensionen werden am signifikantesten beeinflusst, wenn diese für das Erreichen des jeweiligen sektorspezifischen Projektzieles hoch relevant sind. Zweitens, die Teilnahme an Twinning-Aktivitäten kann in Abhängigkeit des Politikfeldes einen signifikanten negativen Effekt auslösen, wenn die Staatsbeamten ausländische Medienerzeugnisse zur politischen Information nutzen (Hypothese 2 b).

Es ist interessant, dass Twinning-Projekte die Einstellungen der Teilnehmer zu der Dimension demokratischen Regierens prägen, die am weitesten mit den sektorspezifischen Zielen der Projekte übereinstimmen. Das Wettbewerbsprojekt beeinflusst signifikant die Einstellungen zu verantwortlichem Regieren. Dies spiegelt das Ziel des Projektes wider, gerichtliche und administrative Verfahren einzuführen, die ein Wettbewerbskontrollsystem garantieren sollen, das mit dem der EU-Mitgliedstaaten vergleichbar ist. Zu diesem Zweck betont das Projekt vor allem die Aktivierung und Stärkung des Wettbewerbsrates in Richtung einer unabhängigen Kontrollautorität. Es versucht weiter »[to] ensure the right of appeal to independent courts against antitrust decisions« (Europäische Kommission 2004: 23) und ein Gerichtssystem mit kompetenten Gerichtshöfen zu garantieren, welches über die von den Wettbewerbsbehörden getroffenen Entscheidungen wacht. Das Twinning-Projekt zur Gesundheitskontrolle von Lebensmitteln für Konsumenten betont insbesondere Partizipation. Es beabsichtigt, den Verbraucherschutz zu stärken, indem Verbraucherverbände und Produzenten verstärkt in den Prozess der Entscheidungsfindung und -durchsetzung von Produktqualitäten mit einbezogen werden. Dieses Projekt ist besonders erfolgreich darin, die Einstellung zu partizipatorischem Regieren signifikant zu beeinflussen. Das Twinning-Projekt zu Umwelt legt besonderen Wert auf die Einrichtung von "procedures concerning access to information and public participation « (Europäische Kommission 2004: 36) und versucht, ein Gesetz zum Zugang zu Umweltinformationen einzuführen. Es beeinflusst am signifikantesten die Einstellungen der Staatsbeamten zu transparentem und partizipatorischem Regieren. 
Die beschriebenen sektoralen Effekte sind keine unabhängigen Effekte, sondern bedürfen eines vorherigen Kennenlernens demokratischen Regierens durch einen Auslandsaufenthalt in westlichen Demokratien. Es scheint als reiche weder die persönliche praktische Erfahrung in westlichen Demokratien noch die Teilnahme an Twinning-Aktivitäten allein, um Staatsbeamte in demokratischem Regieren zu sozialisieren. Vielmehr bedarf es anscheinend sowohl der persönlichen Erfahrung demokratisch administrativen Regierens wie es in westlichen Demokratien praktiziert wird als auch der Wissensvermittlung in Politikreformprojekten, um Einstellungen signifikant zu beeinflussen. Eine Ausnahme ist das Umweltprojekt, das einen signifikanten unabhängigen Effekt auf die Einstellungen der Staatsbeamten zu partizipatorischem Regieren hat. Das Twinning-Projekt zu Zöllen dagegen ruft keinen signifikanten demokratisierenden Effekt hervor, obwohl es besonderen Wert auf verantwortliches Regieren im Hinblick auf interne Prüfungen und der Garantie korrekter und angemessener administrativer Verfahren innerhalb der Behörde für Zölle und indirekte Steuern legt.

Tabelle 4: Vergleich der Netzwerke

\begin{tabular}{|c|c|c|c|c|}
\hline & Zölle & Wettbewerb & $\begin{array}{l}\text { Gesundheits- } \\
\text { kontrolle }\end{array}$ & Umwelt \\
\hline \multicolumn{5}{|c|}{ Regressionsergebnisse für individuelle Twinning-Projekte (vgl. Tabelle IV im Anhang) } \\
\hline $\begin{array}{l}\text { Effekt } \\
\text { (Dimension) }\end{array}$ & $\begin{array}{l}\text { Kein signifikan- } \\
\text { ter Effekt }\end{array}$ & $\begin{array}{l}\text { Signifikanter } \\
\text { bedingter Effekt } \\
\text { (Verantwort- } \\
\text { lichkeit) }\end{array}$ & $\begin{array}{l}\text { Signifikanter } \\
\text { bedingter Effekt } \\
\text { (Partizipation) }\end{array}$ & $\begin{array}{l}\text { Signifikanter be- } \\
\text { dingter (Transpa- } \\
\text { renz). Signifi- } \\
\text { kanter unabhän- } \\
\text { giger Effekt (Par- } \\
\text { tizipation) }\end{array}$ \\
\hline \multicolumn{5}{|c|}{ Eigenschaften der individuellen Twinning-Projekte } \\
\hline Größe & $\begin{array}{l}\text { Groß } \\
\text { (2 Ministerien, } \\
4 \text { Abteilungen) }\end{array}$ & $\begin{array}{l}\text { Klein } \\
\text { (1 Ministerium, } \\
1 \text { Abteilung) }\end{array}$ & $\begin{array}{l}\text { Mittel } \\
\text { (1 Ministerium, } \\
2 \text { Abteilungen } \\
\text { plus Provinz- } \\
\text { Abteilungen) }\end{array}$ & $\begin{array}{l}\text { Mittel } \\
\text { (1 Ministerium, } \\
5 \text { Abteilungen) }\end{array}$ \\
\hline Dauer & $\begin{array}{l}\text { Lang } \\
\text { (18 Monate) }\end{array}$ & $\begin{array}{l}\text { Kurz } \\
\text { (9 Monate) }\end{array}$ & $\begin{array}{l}\text { Mittel } \\
\text { (13 Monate) }\end{array}$ & $\begin{array}{l}\text { Lang } \\
\text { (19 Monate) }\end{array}$ \\
\hline Politisierung & Hoch & Hoch & Mittel & Niedrig \\
\hline
\end{tabular}

Inwiefern können neben dem Sektor die Netzwerkeigenschaften Aufschluss über das unterschiedliche Demokratisierungspotential der einzelnen Projekte geben? Tabelle 4 fasst die vergleichende Analyse zusammen. Es scheint, als ob die Herausbildung von Vertrauen unter den Teilnehmern entscheidend dafür ist, ob ein Netzwerk 
die Einstellungen seiner Teilnehmer prägen kann. ${ }^{28}$ Vertrauen ist wichtig, um einen offenen Austausch zu ermöglichen, in dem auch heiklere Fragen wie der Zugang zu Informationen für Journalisten angesprochen und diskutiert werden können. Es zeigt sich zum einen, dass demokratische Sozialisation erleichtert wird, wenn die Interaktion in einem weniger politisierten Umfeld stattfindet wie beispielweise im Bereich der Umwelt. Zum anderen können Teilnehmer anscheinend auch in politisierten Bereichen wie der Wettbewerbspolitik möglicherweise durch eine besonders konzentrierte Interaktion in demokratische Prinzipien und Praktiken sozialisiert werden.

Während das Twinning-Projekt zu Zöllen (hoher Grad an Politisierung) überhaupt keinen signifikanten demokratisierenden Effekt auszulösen vermag, übt das Umweltprojekt (niedriger Grad an Politisierung) einen signifikanten, unabhängigen Einfluss auf Einstellungen zu demokratischem, partizipatorischem Regieren aus. Interviews vor Ort mit Beteiligten sowie eigene Beobachtungen bestätigten, dass in diesen Bereichen die Teilnehmer mehr Handlungsspielraum geniessen und externen Akteuren weniger misstrauisch begegnet wird. Findet Interaktion jedoch in einem politisierten Umfeld statt, kann ein Netzwerk, das nur eine kleine Anzahl an Teilnehmern umfasst, anscheinend immer noch eine intensive und vertrauenswürdige Interaktion gewährleisten und somit demokratische Sozialisation ermöglichen. Das Twinning-Projekt zu Wettbewerb ist trotz des hohen Grades an Politisierung erfolgreich, seine Teilnehmer in demokratisches Regieren zu sozialisieren. Berichte der Teilnehmer machten hier deutlich, dass sie sich gegenseitig als Menschen kennenlernten und Vertrauen zueinander gewannen. Die Dauer des Projektes, das heißt die Länge des Kontaktes, scheint keinen bedeutenden Einfluss auf die Möglichkeit zu haben, die demokratische Denkweise der Teilnehmer zu beeinflussen. Während die Teilnahme an dem TwinningProjekt zu Zöllen keinen signifikanten Einfluss trotz der langen Dauer generiert, reichte die kurze Dauer des Twinning-Projektes zu Wettbewerb aus, um die Einstellungen der teilnehmenden Staatsbeamten zu demokratischem Regieren zu prägen, wenn diese zuvor im demokratischen Ausland waren.

Die Regressionsanalyse zeigt ausserdem an, dass eine Teilnahme an Twinning-Aktivitäten einen signifikanten negativen Effekt generieren kann, wenn Staatsbeamte ausländische Medien zur politischen Information nutzen. Dies trifft jedoch nur auf Staatsbeamte zu, die an den Projekten zu Wettbewerb oder Gesundheitskontrolle teilgenommen haben. Staatsbeamte, die in das Umweltprojekt involviert waren, wurden dagegen positiv durch die Teilnahme beeinflusst, wenn sie ausländische Medien nutzen (siehe Anhang IV). Während in den Bereichen Wettbewerb und Umwelt die Einstellungen zu demokratischem Regieren insgesamt negativ (Wettbewerb) beziehungsweise positiv (Umwelt) durch Nutzung ausländischer Medien beeinflusst wurden, prägt sie im Bereich Gesundheitskontrolle nur die Einstellung zu dem Aspekt der Transparenz von demokratischem Regieren.

Ein Blick auf Tabelle 4 deutet auf die mögliche Erklärung hin, dass der Grad der Politisierung des Politikbereiches bestimmt, wie die durch ausländische Medien ge-

$28 \mathrm{Zu}$ Vertrauen in professionellen Netzwerken gibt es eine recht umfassende Literatur, siehe beispielsweise Fox (1974: 362); Kramer (1999); Rhodes (2006: 18). 
lieferten Informationen zu demokratischem Regieren wahrgenommen werden. Es scheint, dass demokratische Formen des Regierens in vergleichsweise wenig politisierten Feldern wie Umwelt nicht als Bedrohung für die Autorität des Regimes wahrgenommen werden. In politisch sensiblen Bereichen dagegen ist es wahrscheinlicher, dass demokratisches Regieren als eher beunruhigend wahrgenommen wird von Staatsbeamten, die in autoritären Regimen dienen. Dies vermag vielleicht auch zu erklären, warum die Berichterstattung ausländischer Medien die Abneigung von Staatsbeamten insbesondere gegenüber transparentem Regieren erhöht, die im Bereich der Gesundheitskontrolle arbeiten. Sie könnten befremdet davon sein, wie weit Transparenz gehen kann, wenn sogar internes Regierungsmaterial der Öffentlichkeit zur Verfügung gestellt wird, und welche Konsequenzen eine solche Praxis haben könnte, wenn es in Marokko zur Anwendung käme. Diese Interpretationen sind jedoch nur sehr tentativ und bedürfen weiterer Untersuchungen.

\section{Schlussbetrachtung}

Dieser Artikel untersuchte das demokratisierende Potential von funktionaler Kooperation zwischen den Verwaltungen etablierter Demokratien und autoritärer Regime. Konkret wurde die Frage erörtert, inwiefern ENP-Twinning-Projekte als eine Form funktionaler Kooperation die Einstellungen von involvierten Staatsbeamten zu demokratischem Regieren infolge gemeinsamen Problemlösens und sozialer Interaktion positiv prägen. Die Ergebnisse sind sowohl für die praktische Politikgestaltung als auch für die akademische Forschung relevant. Sie bieten nicht nur Raum für eine optimistischere Einschätzung der Effekte funktionaler Kooperation, indem sie aufzeigen, dass diese subtile Demokratisierungsprozesse auslösen kann, sondern zweifeln auch die bisher vorwiegend negativen Befunde der bisherigen Forschung zu Sozialisationseffekten in und durch internationale Institutionen an.

In theoretischer Hinsicht ist die vorliegende Arbeit vor allem für zwei Literaturstränge relevant: Forschung zu Sozialisationsprozessen in internationalen Kontexten und zur Diffusion transnationaler Normen. Sie spricht zum einen zur Sozialisationsforschung, indem sie internationale Sozialisationsprozesse in einem Kontext untersucht, in dem ein konfundierender Effekt durch nationale Sozialisation weitgehend ausgeschlossen werden kann, beziehungsweise der Einfluss nationaler Sozialisation dem hier untersuchten Effekt transnationaler Sozialisation entgegenwirken sollte. Dadurch dass in der vorliegenden Studie ein Effekt funktionaler Kooperation auf die Einstellungen von staatlichen Beamten zu demokratischem Regieren nachgewiesen werden konnte, stellt sich die Frage, ob nicht auch in anderen Kontexten, inklusive den bereits untersuchten wie der Europäischen Kommission und UN-Versammlungen, doch subtile Prozesse des Einstellungswandels zu transnationalen Normen stattfinden - und die Sozialisationsforschung damit alles andere als ein »erschöpftes« Forschungsprogramm ist (Schimmelfennig 2003: 401; Pollack 1998). Zum anderen bereicherte die vorliegende Arbeit auch die existierende Forschung zur Diffusion transnationaler Normen. Sie führt mit transgouvernementalen Netzwerken nicht nur 
einen bisher vernachlässigten Übermittler transnationaler Normen als empirischen Untersuchungsgegenstand ein, sondern untersucht explizit die Mikrofundierung des Diffusionsarguments. Die negativen Befunde für eine Sozialisierung in demokratischen Prinzipien durch die Nutzung ausländischer Medien sowie Auslandsaufenthalte weckt dabei Zweifel am bisherigen Wissen zu Diffusion von Normen durch transnationalen Austausch.

Konkret liefert die präsentierte Untersuchung vor allem drei Befunde hinsichtlich des demokratisierenden Potentials funktionaler Kooperation im Fall von Marokko: Erstens kann funktionale Kooperation, unter der Bedingung eines vorherigen Aufenthaltes im demokratischen Ausland, Staatsbeamte in demokratischem Regieren sozialisieren. Indem sie die Einstellungen der Teilnehmer zu demokratischen Formen der Entscheidungsfindung signifikant positiv beeinflusst, birgt funktionale Kooperation ein vielversprechendes Potential, die Saat für demokratischen Wandel inmitten autoritärer Regime zu säen. Inwieweit und unter welchen Bedingungen diese Saat, das heißt eine positive Einstellung zu demokratischem Regieren seitens einiger Staatsbeamter, tatsächlich in alltägliche administrative Praktiken umgesetzt wird, bedarf weiterer Studien. Es bleibt ebenso abzuwarten, ob solch demokratisches administratives Regieren letztendlich spill over-Effekte auf allgemeine Regierungsstrukturen auslösen kann, indem es Dynamiken freisetzt, die Demokratisierung (anstelle von Stabilisierung) des gesamten politischen Systems fordern. Im Lichte der jüngsten Ereignisse wäre es zudem interessant zu untersuchen, inwieweit das veränderte politische Umfeld in ursprünglich weniger liberalisierten Staaten wie Tunesien und Ägypten auch hier ähnliche Prozesse ermöglicht. In Ägypten gehörte institutioneller Kapazitätsaufbau zu den Prioritäten der Europäischen Nachbarschaftspolitik. Für den Zeitraum 2004 bis 2010 gibt die Europäische Union an, insgesamt 14 Twinning-Projekte in Bereichen wie Transport, Tourismus und Umwelt entweder bereits abgeschlossen oder noch am Laufen zu haben (Europäische Union 2009). Auch gehört Ägypten zu den ersten Ländern unter den südlichen Mittelmeeranrainern, welche an dem 2008 lancierten und mit der Organisation für Wirtschaftliche Zusammenarbeit und Entwicklung (OECD) gemeinsam durchgeführten Programm zur Unterstützung bei der Verbesserung von Governance und Management (SIGMA, Support for Improvement in Governance and Management) teilnehmen, welches die Reform administrativer Entscheidungsfindungsprozesse in den Mittelpunkt stellt. Die Nachrichten über Staatsbeamte, welche sich an den Protesten zu Beginn des Jahres 2011 in Ägypten beteiligten und teilweise ihre Arbeit kündigten, unterstützt eine derartig optimistische Lesart der Ergebnisse der vorliegenden Studie.

Die Ergebnisse zeigen zweitens, dass demokratische Sozialisation durch sektorale Kooperation in jenen Dimensionen demokratischen Regierens am signifikantesten ist, die am relevantesten für den jeweiligen Sektor sind. Dieser Befund stärkt das funktionale Argument von demokratischer Sozialisation als einem Nebeneffekt technischer Problemlösung. Insbesondere die Analyse des Twinning-Projektes zur Umwelt zeigt, dass ein Verweis auf demokratische Elemente während der gemeinsamen Politikentwicklung zu einem Transfer demokratischer Normen durch funktionale Kooperation führen kann. Es wäre interessant zu untersuchen, inwieweit dieser Be- 
fund über das EU-Twinning-Programm hinaus auf andere Politikreformprogramme verallgemeinert werden kann. Wichtig ist jedoch, dass funktionale Kooperation mit der Intention von Demokratisierung ihre politische Unschuld verliert und damit vermutlich auch ihr Potential, subtile Demokratisierungsprozesse zu initialisieren. Trotz alledem scheint es, als ob die Europäische Kommission ihre Nachbarschaftspolitik in diese Richtung weiter ausbauen möchte. In einem Beitrag in The International Herald Tribune vom 26. Februar 2011 verkündete die Hohe Vertreterin für EU-Außenpolitik Catherine Ashton, dass die Unterstützung der EU »will involve detailed, unglamorous, work on the ground - with civil servants, local communities, the police, army and judiciary - laying the foundations of deep democracy and then building it up, brick-by-brick« (Ashton 2011).

Ein drittes Ergebnis weist darauf hin, dass ein niedriger Politisierungsgrad der Politikbereiche demokratische Sozialisation vereinfacht und dass ein hoher Politisierungsgrad durch intensive Kooperation unter einer kleinen Anzahl an Teilnehmern kompensiert werden kann. Der Befund zur Bedeutung des Politisierungsgrades für demokratische Sozialisation in transgouvernementalen Politiknetzwerken weist auf die Grenzen dieser Möglichkeit der Demokratieförderung hin. Es scheint, als ob funktionale Kooperation in einem Rahmen stattfinden muss, welcher einen offenen Austausch über demokratische Prinzipien und Praktiken demokratischer Entscheidungsfindung erlaubt. Es bedarf jedoch weiterer Studien, um genauer sagen zu können, inwieweit nicht nur die Qualität sozialer Interaktion, sondern auch der allgemeine Grad politischer Liberalisierung die Wahrscheinlichkeit einer Sozialisation in demokratisches Regieren durch funktionale Kooperation erhöht beziehungsweise verringert.

Diese Studie möchte weitere Forschung zu diesen Fragen anstoßen. Sie bietet eine erste Analyse des demokratisierenden Potentials funktionaler Kooperation - einer Kooperation, die von den autoritären Eliten angefragt wird und die Raum für einen intensiven Austausch zwischen westlichen Demokratien und autoritären Regimen bietet. Es erscheint lohnenswert, subtile Prozesse der Demokratisierung, die durch funktionale Kooperation generiert werden, weiter zu erforschen. Schließlich sind alle Instrumente und Strategien (mit Ausnahme militärischer Eingriffe), welche von externen Akteuren im Rahmen ihrer direkten Demokratieförderungspolitiken gegenüber stabilen autoritären Regimen angewandt werden, zum Scheitern verurteilt, wenn die herrschende Elite wenig Neigung zeigt, ihre Macht zu beschränken.

\section{Literatur}

Al-Arkoubi, Khadija/McCourt, Willy 2004: The Politics of HRM: Waiting for Godot in the Moroccan Civil Service, in: International Journal of Human Resource Management 15: 6, 978-995.

Albrecht, Holger/Schlumberger, Oliver 2004: »Waiting for Godot «: Regime Change without Democratization in the Middle East, in: International Political Science Review 25: 4, 371-392. 
Ashton, Catherine 2011: Listening to the Revolution, in: The International Herald Tribune, 26.02.2011.

Atkinson, Carol 2010: Does Soft Power Matter? A Comparative Analysis of Student Exchange Programs 1980-2006, in: Foreign Policy Analysis 6: 1, 1-22.

Baker, Randall (Hrsg.) 2002: Transitions from Authoritarianism: The Role of the Bureaucracy, London.

Beetham, David 1999: Democracy and Human Rights, Cambridge.

Berger, Morroe 1957: Bureaucracy and Society in Modern Egypt: A Study of the Higher Civil Service, Princeton, NJ.

Beyers, Jan 2005: Multiple Embeddedness and Socialization in Europe: The Case of Council Officials, in: International Organization 59: 4, 899-936.

Bovens, Mark 2007: New Forms of Accountability and EU-Governance, in: Comparative European Politics 5: 1, 104-120.

Brambor, Thomas/Clark, William Roberts/Golder, Matt 2006: Understanding Interaction Models: Improving Empirical Analyses, in: Political Analysis 14: 1, 63-82.

Braumoeller, Bear F. 2004: Hypothesis Testing and Multiplicative Interaction Terms, in: International Organization 58: 3, 807-820.

Brinkerhoff, Derick W. 2000: Democratic Governance and Sectoral Policy Reform: Tracing Linkages and Exploring Synergies, in: World Development 28: 4, 601-615.

Brown, Timothy A. 2006: Confirmatory Factor Analysis for Applied Research, New York, NY.

Brownlee, Jason 2007: Authoritarianism in an Age of Democratization, Cambridge.

Burnell, Peter/Schlumberger, Oliver 2010: Promoting Democracy - Promoting Autocracy? International Politics and National Political Regimes, in: Contemporary Politics 16: 1, 1-15.

Campbell, Donald T./Stanley, Julian C. 1966: Experimental and Quasi-Experimental Designs for Research, Chicago, IL.

Campbell, Patricia J. 2003: Morocco in Transition: Overcoming the Democratic and Human Rights Legacy of King Hassan II., in: African Studies Quarterly 7: 1, 38-58.

Checkel, Jeffrey T. 2001: Why Comply? Social Learning and European Identity Change, in: International Organization 55: 3, 553-588.

Checkel, Jeffrey T. 2003: »Going Native« in Europe? Theorizing Social Interaction in European Institutions, in: Comparative Political Studies 36: 1/2, 209-231.

Checkel, Jeffrey T. 2005: International Institutions and Socialization in Europe: Introduction and Framework, in: International Organization 59: 4, 801-826.

Collins, Debbie 2003: Pretesting Survey Instruments: An Overview of Cognitive Methods, in: Quality of Life Research 12: 3, 229-238.

Cooper, Chris/Johansen, Mikael 2003: An Evaluation of Completed Twinning Projects, (Bericht für das Treffen der National Contact Points), Brüssel.

Coston, Jennifer M. 1998: Administrative Avenues to Democratic Governance: The Balance of Supply and Demand, in: Public Administration and Development 18: 5, 479-493.

Dahl, Robert A. 1971: Polyarchy: Participation and Opposition, New Haven, CT.

Dawson, Richard E./Prewitt, Kenneth 1969: Political Socialization: An Analytic Study, Boston, MA.

Desrues, Thierry/Moyano, Eduardo 2001: Social Change and Political Transition in Morocco, in: Mediterranean Politics 6: 1, 21-47.

Diamond, Larry/Plattner, Marc F./Schedler, Andreas 1999: Introduction, in: Schedler, Andreas/ Diamond, Larry/Plattner, Marc F. (Hrsg.): The Self-Restraining State: Power and Accountability in New Democracies, Boulder, CO, 1-10.

Europäische Kommission 2004: EU/Morocco Action Plan, Annex to Proposal for a Council Decision on the Position To Be Adopted by the European Community and Its Member States $[\ldots]$ on the Implementation of the EU-Morocco Action Plan (COM(2004) 788 final), Brüssel. 
Europäischer Rechnungshof 2003: Special Report $n^{\circ}$ 6/2003 Concerning Twinning as a Main Instrument To Support Institution-Building in Candidate Countries, Together with the Commission's Replies, in: Official Journal C167, 21-45.

Europäische Union 2009: European Neighborhood Policy - Egypt MEMO/09/179, in: http:// europa.eu/rapid/pressReleasesAction.do?reference=MEMO/09/179\&format=HTML\&ag $\mathrm{ed}=0$ \&language $=$ EN\& guiLanguage $=\mathrm{en} ; 14.03 .2011$.

Fearon, James/Wendt, Alexander 2003: Rationalism versus Constructivism: A Skeptical View, in: Carlsnaes, Walter/Risse, Thomas/Simmons, Beth A. (Hrsg.): Handbook of International Relations, London, 52-72.

Finnemore, Martha 1993: International Organizations as Teachers of Norms: The United Nations Educational, Scientific, and Cultural Organization and Science Policy, in: International Organization 47: 4, 565-597.

Flockhart, Trine 2004: »Masters and Novices«: Socialization and Social Learning through the NATO Parliamentary Assembly, in: International Relations 18: 3, 361-380.

Fox, Alan 1974: Beyond Contract: Work, Power and Trust Relations, London.

Freyburg, Tina/Lavenex, Sandra/Schimmelfennig, Frank/Skripka, Tatiana/Wetzel, Anne 2009: EU Promotion of Democratic Governance in the Neighbourhood, in: Journal of European Public Policy 16: 6, 916-934.

Freyburg, Tina/Skripka, Tatiana/Wetzel, Anne 2007: Democracy between the Lines? EU Promotion of Democratic Governance via Sector-Specific Co-Operation (NCCR Democracy Working Paper 5), Zürich.

Gandhi, Jennifer/Przeworski, Adam 2007: Authoritarian Institutions and the Survival of Autocrats, in: Comparative Political Studies 40: 11, 1279-1301.

Gheciu, Alexandra 2005: Security Institutions as Agents of Socialization? NATO and the »New Europe«, in: International Organization 59: 4, 973-1012.

Göbel, Christian/Lambach, Daniel 2009: Accounting for the (In-)Stability of Authoritarian Regimes: Evidence from East Asia and Sub-Saharan Africa (Manuskript für die Jahreskonferenz der APSA, Toronto, 03.-06.09.2009).

Grant, Ruth W./Keohane, Robert O. 2005: Accountability and Abuses of Power in World Politics, in: American Political Science Review 99: 1, 29-43.

Harders, Cilja 2008: Analyzing Regional Cooperation after September 11, 2001: The Emergence of a New Regional Order in the Arab World, in: Harders, Cilja/Legrenzi, Matteo (Hrsg.): Beyond Regionalism? Regional Cooperation, Regionalism and Regionalization in the Middle East, Aldershot, 33-50.

Holland, Paul W. 1986: Statistics and Causal Inference, in: Journal of the American Statistical Association 81: 396, 945-960.

Hooghe, Liesbet 2005: Several Roads Lead to International Norms, but Few via International Socialization: A Case Study of the European Commission, in: International Organization 59: 4, 861-898.

Hyden, Goran/Court, Julius/Mease, Kenneth 2004: Making Sense of Governance: Empirical Evidence from Sixteen Developing Countries, Boulder, CO.

John, Oliver P./Benet-Martínez, Veronica 2000: Measurement: Reliability, Construct Validation, and Scale Construction, in: Reis, Harry T./Jud, Charles M. (Hrsg.): Handbook of Research Methods in Social and Personality Psychology, Cambridge, 339-369.

Johnston, Alaistair Iain 2001: Treating International Institutions as Social Environments, in: International Studies Quarterly 45: 4, 487-515.

Johnston, Alaistair Iain 2005: Conclusions and Extensions: Toward Mid-Range Theorizing and Beyond Europe, in: International Organization 59: 4, 1013-1044.

Kaufmann, Daniel/Kraay, Aart/Mastruzzi, Massimo 2005: Governance Matters IV: Governance Indicators for 1996-2004 (The World Bank Policy Research Working Paper 3630), Washington, DC.

Keohane, Robert O./Nye, Joseph S. 1974: Transgovernmental Relations and International Organizations, in: World Politics 27: 1, 39-62. 
Kern, Holger Lutz/Hainmueller, Jens 2009: Opium for the Masses: How Foreign Media Can Stabilize Authoritarian Regimes, in: Political Analysis 17: 4, 377-399.

Kerr, Henry H. 1973: Changing Attitudes through International Participation: European Parliamentarians and Integration, in: International Organization 27: 1, 45-83.

Kramer, Roderick M. 1999: Trust and Distrust in Organizations: Emerging Perspectives, Enduring Questions, in: Annual Review of Psychology 50, 569-598.

Leib, Ethan/He Baogang (Hrsg.) 2006: The Search for Deliberative Democracy in China, New York, NY.

Marsden, Peter V. 1990: Network Data and Measurement, in: Annual Review of Sociology 16, 435-463.

Marsh, David 1971: Political Socialization: The Implicit Assumptions Questioned, in: British Journal of Political Science 1: 4, 453-465

Mohamedou, Mohammad-Mahmoud 1999: The Rise and Fall of Democratization in the Maghreb, in: Magnarella, Paul J. (Hrsg.): Middle East and North Africa: Governance, Democratization, Human Rights, Aldershot, 209-239.

Moravcsik, Andrew 1993: Preferences and Power in the European Community: A Liberal Intergovernmentalist Approach, in: Journal of Common Market Studies 31: 4, 473-524.

Morse, Janice M. 2002: Principles of Mixed Methods and Multimethod Research Design, in: Tashakkori, Abbas/Teddlie, Charles (Hrsg.): Handbook of Mixed Methods in Social and Behavioral Research, Thousand Oaks, CA, 189-208.

Muthén, Linda K./Muthén, Bengt O. 2006: Mplus User's Guide, 4. Auflage, Los Angeles, CA.

Nye, Joseph S. 2004: Soft Power: The Means to Success in World Politics, New York, NY.

Organisation für Wirtschaftliche Zusammenarbeit und Entwicklung (OECD) 2011: Morocco, in: http://www.oecd.org/dataoecd/23/6/1882353.gif; 11.03.2011.

Page, Edward C. 1985: Political Authority and Bureaucratic Power. A Comparative Analysis, Brighton.

Papadimitriou, Dimitris/Phinnemore, David 2003: Exporting Europeanization to the Wider Europe: The Twinning Exercise and Administrative Reform in the Candidate Countries and Beyond, in: Southeast European and Black Sea Studies 3: 2, 1-22.

Pawelka, Peter 2002: Der Staat im Vorderen Orient: Über die Demokratie-Resistenz in einer globalisierten Welt, in: Leviathan 30: 4, 432-454.

Pérez-Armendáriz, Clarisa/Crow, David 2010: Do Migrants Remit Democracy? International Migration, Political Beliefs, and Behavior in Mexico, in: Comparative Political Studies 43: 1, 119-148.

Pilotte, William/Gable, Robert K. 1990: The Impact of Positive and Negative Item Stems on the Validity of a Computer Anxiety Scale, in: Educational and Psychological Measurement 50: 3, 603-610.

Pollack, Mark A. 1998: Constructivism, Social Psychology, and Elite Attitude Change: Lessons from an Exhausted Research Program (Manuskript für die 11. Konferenz der Europawissenschaftler, Baltimore, MD, 26.02.-01.03.1998).

Pollack, Mark A. 2005: The New Transatlantic Agenda at Ten: Reflections on an Experiment in International Governance, in: Journal of Common Market Studies 43: 5, 899-919.

Presser, Stanley/Couper, Mick P./Lessler, Judith T./Martin, Elizabeth/Martin, Jean/Rothgeb, Jennifer M./Singer, Eleanor 2004: Methods for Testing and Evaluating Survey Questions, in: Public Opinion Quarterly 68: 1, 109-130.

Raykov, Tenko 2007: Reliability if Deleted, Not »Alpha if Deleted«: Evaluation of Scale Reliability Following Component Deletion, in: British Journal of Mathematical and Statistical Psychology 60: 2, 201-216.

Rhodes, Roderick A.W. 2006: The Sour Laws of Network Governance, in: Fleming, Jenny/Dawn Wood, Jennifer (Hrsg.): Fighting Crime Together: The Challenges of Policing and Security Networks, Sydney, 15-35.

Risse, Thomas/Ropps, Stephen C./Sikkink, Kathryn (Hrsg.) 1999: The Power of Human Rights: International Norms and Domestic Change, Cambridge. 
Schimmelfennig, Frank 2003: Internationale Sozialisation: Von einem »erschöpften« zu einem produktiven Forschungsprogramm? in: Hellmann, Gunther/Wolf, Klaus Dieter/Zürn, Michael (Hrsg.): Die neuen internationalen Beziehungen. Forschungsstand und Perspektiven in Deutschland, Baden-Baden, 401-427.

Schimmelfennig, Frank/Engert, Stefan/Knobel, Heiko 2006: International Socialization in Europe, Basingstoke.

Schlumberger, Oliver 2006: Dancing with Wolves: Dilemmas of Democracy Promotion in Authoritarian Contexts, in: Jung, Dieter (Hrsg.): Democratization and Development: New Political Strategies for the Middle East, Basingstoke, 33-60.

Schriesheim, Chester A./Eisenbach, Regina J./Hill, Kenneth D. 1991: The Effect of Negation and Polar Opposite Item Reversals on Questionnaire Reliability and Validity: An Experimental Investigation, in: Educational and Psychological Measurement 51: 1, 67-78.

Scully, Roger 2005: Becoming Europeans? Attitudes, Behaviour, and Socialization in the European Parliament, Oxford.

Searing, Donald/Wright, Gerald/Rabinowitz, George 1976: The Primacy Principle: Attitude Change and Political Socialization, in: British Journal of Political Science 6: 1, 83-113.

Sijtsma, Klaas 2009: On the Use, the Misuse, and the Very Limited Usefulness of Cronbach's Alpha, in: Psychometrika 74: 1, 107-120.

Slaughter, Anne-Marie 2000: Government Networks: The Heart of the Liberal Democratic Order, in: Fox, Gregory H./Roth, Brad R. (Hrsg.): Democratic Governance and International Law, Cambridge, 199-235.

Slaughter, Anne-Marie 2004: A New World Order, Princeton, NJ.

United States Agency for International Development (USAID) 2011: Morocco, in: http:// www.usaid.gov/pubs/bj2001/ane/ma/; 11.03.2011.

van Waarden, Frans 1992: Dimensions and Types of Policy Networks, in: European Journal of Political Research 21: 1/2, 29-52.

Verba, Sidney 1967: Democratic Participation, in: Annals of the American Academy of Political and Social Science 373: 2, 53-78.

Way, Lucan A./Levitsky, Steven 2007: Linkage, Leverage, and the Post-Communist Divide, in: East European Politics and Societies 21: 1, 48-66.

Wejnert, Barbara 2005: Diffusion, Development, and Democracy 1800-1999, in: American Sociological Review 70: 1, 53-81.

Whitehead, Laurence 1996: Three International Dimensions of Democratization, in: Whitehead, Laurence (Hrsg.): The International Dimensions of Democratization: Europe and the Americas, Oxford, 3-25.

Worthington, Roger L./Whittaker, Tiffany A. 2006: Scale Development Research: A Content Analysis and Recommendations for Best Practices, in: The Counseling Psychologist 34: 6, 806-38.

Youngs, Richard 2001: European Union Democracy Promotion Policies: Ten Years On, in: European Foreign Affairs Review 6: 3, 355-373.

Zaharchenko, Tatiana R./Goldenman, Gretta 2004: Accountability in Governance: The Challenge of Implementing the Aarhus Convention in Eastern Europe and Central Asia, in: International Environmental Agreements: Politics, Law and Economics 4: 3, 229-251.

Zerhouni, Saloua 2004: Morocco: Reconciling Continuity and Change, in: Perthes, Volker (Hrsg.): Arab Elites: Negotiating the Politics of Change, Boulder, CO, 61-85. 
Anhang

Ia: Deskriptive Statistiken - Abhängige Variablen

\begin{tabular}{lcccc}
\hline & Partizipation & Transparenz & Verantwortlichkeit & Demokratisches Regieren \\
\hline Max. Wert & 5.00 & 5.00 & 5.00 & 5.00 \\
Min. Wert & 2.50 & 2.25 & 2.00 & 2.83 \\
AM & 4.30 & 4.04 & 3.84 & 4.06 \\
Median & 4.50 & 4.00 & 4.00 & 4.11 \\
SA & .578 & .427 & .672 & .382 \\
Schiefe & -.751 & -.547 & -.635 & -.570 \\
\hline
\end{tabular}

Deskriptive Statistiken; Werte rangieren zwischen 1 (nichtdemokratisch) und 5 (demokratisch); $N=110$, Fälle mit fehlenden Werten listenweise ausgeschlossen; AM=Arithmetisches Mittel, SA=Standardabweichung.

Ib: Deskriptive Statistiken - Unabhängige Variablen

\begin{tabular}{|c|c|c|c|c|c|}
\hline & Kooperation & $\begin{array}{l}\text { Admin. Sozia- } \\
\text { lisation }\end{array}$ & $\begin{array}{l}\text { Auslands- } \\
\text { aufenthalt }\end{array}$ & $\begin{array}{l}\text { Ausländ. } \\
\text { Medien }\end{array}$ & $\begin{array}{c}\text { Andere Pro- } \\
\text { gramme }\end{array}$ \\
\hline Arithmetisches Mittel & .56 & .61 & .42 & .79 & .53 \\
\hline Median & 1 & 1 & 0 & 1 & 1 \\
\hline \multirow[t]{2}{*}{ Häufigkeiten } & 44.4 & 61.5 & 57.8 & 20.7 & 46.7 \\
\hline & 55.6 & 38.5 & 42.2 & 79.3 & 53.3 \\
\hline Standardabweichung & .407 & .488 & .496 & .407 & .501 \\
\hline (1) Kooperation & 1.00 & & & & \\
\hline (2) Admin. Sozialisation & -.018 & 1.00 & & & \\
\hline (3) Auslandsaufenthalt & .051 & $.170^{*}$ & 1.00 & & \\
\hline (4) Ausländische Medien & $.201 * *$ & $.194 *$ & .071 & 1.00 & \\
\hline (5) Andere Programme & .080 & $.158^{*}$ & $.158^{*}$ & $.198 * *$ & 1.00 \\
\hline
\end{tabular}

Deskriptive Statistiken; Häufigkeiten in Prozent; $N=135$, Fälle mit fehlenden Werten listenweise ausgeschlossen; einseitige $p$-Werte der nichtparametrischen Spearman-Rho-Koeffizienten; ${ }^{*} \leq \leq 05, *^{*} p \leq 01$. 


\section{II: Ergebnisse der explorativen Faktorenanalyse}

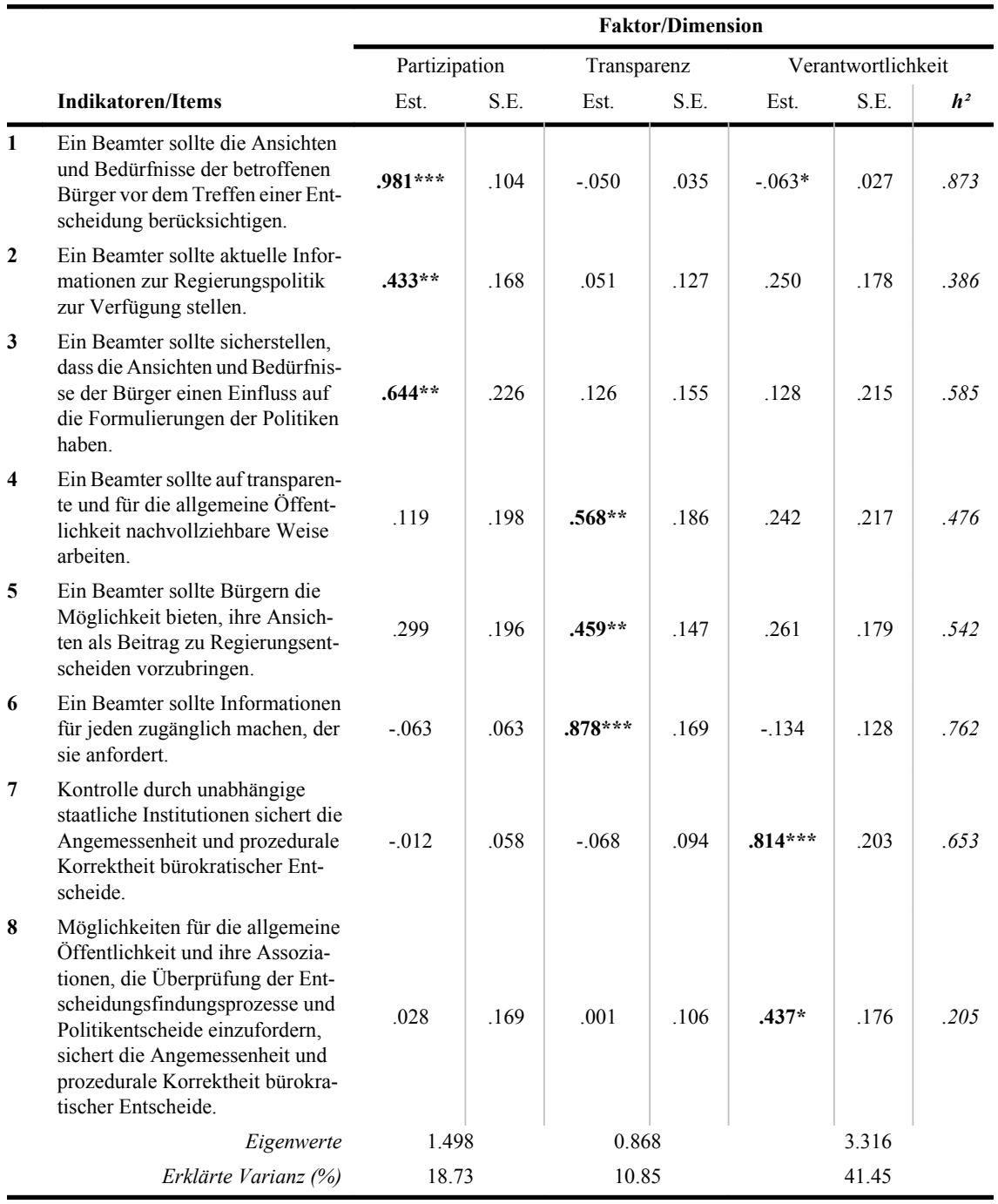

Matrix der Faktorladungen; $N=148$, Est. $=$ Faktorladung (Schätzer), S.E. $=$ Standardfehler, $h^{2}=$ Kommunalität; Faktorenladungen $>.40$ sind fett markiert; ${ }^{*} p \leq .05, * * p \leq .01, * * * p \leq .001$. 
III: Regressionsergebnisse für die individuellen Dimensionen demokratischen Regierens

\begin{tabular}{|c|c|c|c|c|c|c|}
\hline & & (1) & (2) & (3a) & (3 b) & (4) \\
\hline \multirow{11}{*}{ 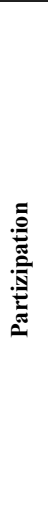 } & Kooperation & $.195(.102)$ & $.194(.105)^{\dagger}$ & $.004(.977)$ & $.437(.168)^{* *}$ & \\
\hline & Admin. Sozialisation & & $-.137(.103)$ & $-.145(.103)$ & $-.145(.103)$ & $-.153(.105)$ \\
\hline & Auslandsaufenthalt & & $-.112(.108)$ & $-.350(.165)^{*}$ & $.350(.165)^{*}$ & $-.092(.108)$ \\
\hline & Ausländ. Medien & & $-.187(.131)$ & $-.137(.129)$ & $-.137(.129)$ & $-.138(.129)$ \\
\hline & Andere Programme & & $.237(.109)^{*}$ & $.228(.107)^{*}$ & $.228(.107)^{*}$ & $.242(.111)^{*}$ \\
\hline & $\begin{array}{l}\text { Koop x 1: Auslands- } \\
\text { aufenthalt }\end{array}$ & & & $.433(.212)^{*}$ & & \\
\hline & Koop x 1: kein & & & & $-.433(.212)^{*}$ & \\
\hline & $R^{2}$ & .028 & .098 & .130 & .130 & .072 \\
\hline & $A I C$ & 426.984 & 1014.939 & 1011.245 & 1011.245 & 848.356 \\
\hline & Log Likelihood & -210.492 & -500.470 & -497.623 & -497.623 & -418.178 \\
\hline & $N$ & 133 & 121 & 121 & 121 & 121 \\
\hline \multirow{9}{*}{ 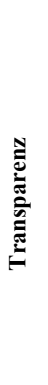 } & Kooperation & $-.113(.075)$ & $-.103(.079)$ & & & \\
\hline & Admin. Sozialisation & & $-.005(.083)$ & & & $.007(.080)$ \\
\hline & Auslandsaufenthalt & & $-.041(.083)$ & & & $-.045(.082)$ \\
\hline & Ausländ. Medien & & $.013(.097)$ & & & $-.014(.094)$ \\
\hline & Andere Programme & & $.204(.080)^{*}$ & & & $.201(.080)^{*}$ \\
\hline & $R^{2}$ & .016 & .071 & & & .056 \\
\hline & $A I C$ & 351.751 & 935.610 & & & 767.177 \\
\hline & Log Likelihood & -172.875 & -460.805 & & & -377.589 \\
\hline & $N$ & 132 & 121 & & & 121 \\
\hline \multirow{9}{*}{ 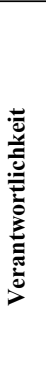 } & Kooperation & $.049(.684)$ & $.004(.128)$ & & & \\
\hline & Admin. Sozialisation & & $-.034(.116)$ & & & $-.034(.116)$ \\
\hline & Auslandsaufenthalt & & $-.072(.121)$ & & & $-.072(.119)$ \\
\hline & Ausländ. Medien & & $.063(.166)$ & & & $.064(.154)$ \\
\hline & Andere Programme & & $.253(.117)^{*}$ & & & $.253(.117)^{*}$ \\
\hline & $R^{2}$ & .001 & .044 & & & .044 \\
\hline & $A I C$ & 437.730 & 994.128 & & & 834.799 \\
\hline & Log Likelihood & -215.865 & -490.064 & & & -411.399 \\
\hline & $N$ & 126 & 117 & & & 117 \\
\hline
\end{tabular}

Multiple Regressionsanalysen (MLMV); Regressionskoeffizienten sind nicht standardisiert; Standardfehler in Klammern; Fälle mit fehlenden Werten listenweise ausgeschlossen; ${ }^{\dagger} p \leq .065,{ }^{*} p \leq .05,{ }^{*} p \leq .01$. 
IVa: Regressionsergebnisse - Das Twinning-Projekt »Zölle»

\begin{tabular}{|c|c|c|c|c|}
\hline & & & (1) & (2) \\
\hline \multirow{9}{*}{ 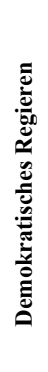 } & Twinning & & $-.142(.122)$ & $-.183(.129)$ \\
\hline & Admin. Sozialisation & & & $-.053(.073)$ \\
\hline & Auslandsaufenthalt & & & $-.055(.074)$ \\
\hline & Ausländ. Medien & & & $-.047(.089)$ \\
\hline & Andere Programme & & & $.232(.077)^{* *}$ \\
\hline & & $R^{2}$ & .013 & .111 \\
\hline & & $A I C$ & 159.791 & 671.043 \\
\hline & & Log Likelihood & -76.895 & -328.522 \\
\hline & & $N$ & 110 & 103 \\
\hline \multirow{9}{*}{ 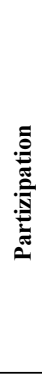 } & Twinning & & $-.246(.221)$ & $-.344(.211)$ \\
\hline & Admin. Sozialisation & & & $-.156(.100)$ \\
\hline & Auslandsaufenthalt & & & $-.095(.105)$ \\
\hline & Ausländ. Medien & & & $-.129(.128)$ \\
\hline & Andere Programme & & & $.247(.108)^{*}$ \\
\hline & & $R^{2}$ & .017 & .103 \\
\hline & & $A I C$ & 299.589 & 897.002 \\
\hline & & Log Likelihood & -146.794 & -441.501 \\
\hline & & $N$ & 133 & 121 \\
\hline \multirow{9}{*}{ 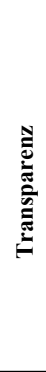 } & Twinning & & $-.234(.145)$ & $-.206(.153)$ \\
\hline & Admin. Sozialisation & & & $.003(.081)$ \\
\hline & Auslandsaufenthalt & & & $-.050(.081)$ \\
\hline & Ausländ. Medien & & & $-.002(.091)$ \\
\hline & Andere Programme & & & $.199(.079)^{*}$ \\
\hline & & $R^{2}$ & .030 & .081 \\
\hline & & $A I C$ & 238.359 & 832.669 \\
\hline & & Log Likelihood & -116.180 & -409.334 \\
\hline & & $N$ & 132 & 121 \\
\hline \multirow{9}{*}{ 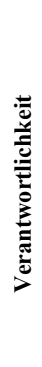 } & Twinning & & $-.037(.144)$ & $-.083(.157)$ \\
\hline & Admin. Sozialisation & & & $-.039(.115)$ \\
\hline & Auslandsaufenthalt & & & $-.075(.118)$ \\
\hline & Ausländ. Medien & & & $.072(.153)$ \\
\hline & Andere Programme & & & $.256(.116)^{*}$ \\
\hline & & $R^{2}$ & .000 & .045 \\
\hline & & $A I C$ & 313.781 & 886.563 \\
\hline & & Log Likelihood & -153.891 & -436.282 \\
\hline & & $N$ & 126 & 117 \\
\hline
\end{tabular}

Multiple Regressionsanalysen (MLMV); Regressionskoeffizienten sind nicht standardisiert; Standardfehler in Klammern; Fälle mit fehlenden Werten listenweise ausgeschlossen; ${ }^{*} p \leq .05$, ** $\leq .01$. 
Tina Freyburg: Demokratisierung durch Zusammenarbeit?

IVb: Regressionsergebnisse - Das Twinning-Projekt »Wettbewerb«

\begin{tabular}{|c|c|c|c|c|c|c|c|}
\hline & & (1) & (2) & (3a) & (3 b) & (3c) & (3d) \\
\hline \multirow{13}{*}{ 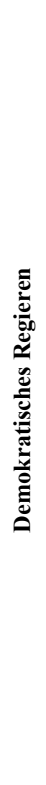 } & Twinning & $\begin{array}{l}.256 \\
(.122)^{*}\end{array}$ & $\begin{array}{l}.207 \\
(.088)^{*}\end{array}$ & $\begin{array}{l}.444 \\
(.098)^{* * *}\end{array}$ & $\begin{array}{l}.161 \\
(.091)^{\dagger}\end{array}$ & $\begin{array}{l}.037 \\
(.097)\end{array}$ & $\begin{array}{l}.037 \\
(.076)^{* * *}\end{array}$ \\
\hline & Admin. Sozialisation & & $\begin{array}{l}-.038 \\
(.074)\end{array}$ & $\begin{array}{l}-.034 \\
(.074)\end{array}$ & $\begin{array}{l}-.034 \\
(.074)\end{array}$ & $\begin{array}{l}-.045 \\
(.074)\end{array}$ & $\begin{array}{l}-.045 \\
(.074)\end{array}$ \\
\hline & Auslandsaufenthalt & & $\begin{array}{l}-.055 \\
(.074)\end{array}$ & $\begin{array}{l}-.062 \\
(.076)\end{array}$ & $\begin{array}{l}-.062 \\
(.076)\end{array}$ & $\begin{array}{l}-.075 \\
(.078)\end{array}$ & $\begin{array}{l}.075 \\
(.078)\end{array}$ \\
\hline & Ausländ. Medien & & $\begin{array}{l}-.068 \\
(.089)\end{array}$ & $\begin{array}{l}-.055 \\
(.091)\end{array}$ & $\begin{array}{l}.055 \\
(.091)\end{array}$ & $\begin{array}{l}-.053 \\
(.089)\end{array}$ & $\begin{array}{l}-.053 \\
(.089)\end{array}$ \\
\hline & Andere Programme & & $\begin{array}{l}.230 \\
(.077)^{* *}\end{array}$ & $\begin{array}{l}.233 \\
(.077)^{* *}\end{array}$ & $\begin{array}{l}.233 \\
(.077)^{* *}\end{array}$ & $\begin{array}{l}.231 \\
(.077)^{* *}\end{array}$ & $\begin{array}{l}.231 \\
(.077)^{* *}\end{array}$ \\
\hline & $\begin{array}{l}\text { Twin. x 1: ausländ. Me- } \\
\text { dien }\end{array}$ & & & $\begin{array}{l}-.283 \\
(.130)^{*}\end{array}$ & & & \\
\hline & $\begin{array}{l}\text { Twin. } \times 1 \text { : keine aus- } \\
\text { länd. Medien }\end{array}$ & & & & $\begin{array}{l}.283 \\
(.130)^{*}\end{array}$ & & \\
\hline & $\begin{array}{l}\text { Twin. } \mathrm{x} 1 \text { : Auslandsauf- } \\
\text { enthalt }\end{array}$ & & & & & $\begin{array}{l}.341 \\
(.125)^{* *}\end{array}$ & \\
\hline & $\begin{array}{l}\text { Twin. } \mathrm{x} \text { 1: kein Aus- } \\
\text { landsaufenthalt }\end{array}$ & & & & & & $\begin{array}{l}-.341 \\
(.125)^{* *}\end{array}$ \\
\hline & $R^{2}$ & .027 & .105 & .110 & .110 & .116 & .116 \\
\hline & $A I C$ & 104.445 & 615.834 & 405.490 & 405.490 & 464.593 & 464.593 \\
\hline & Log Likelihood & -49.222 & -300.917 & -194.745 & -194.745 & -224.296 & -224.296 \\
\hline & $N$ & 110 & 103 & 103 & 103 & 103 & 103 \\
\hline \multirow{9}{*}{ } & Twinning & $\begin{array}{l}.150 \\
(.124)\end{array}$ & $\begin{array}{l}.188 \\
(.154)\end{array}$ & & & & \\
\hline & Admin. Sozialisation & & $\begin{array}{l}-.144 \\
(.105)\end{array}$ & & & & \\
\hline & Auslandsaufenthalt & & $\begin{array}{l}-.095 \\
(.108)\end{array}$ & & & & \\
\hline & Ausländ. Medien & & $\begin{array}{l}-.147 \\
(.130)\end{array}$ & & & & \\
\hline & Andere Programme & & $\begin{array}{l}.254 \\
(.112)^{*}\end{array}$ & & & & \\
\hline & $R^{2}$ & .004 & .077 & & & & \\
\hline & $A I C$ & 233.461 & 837.395 & & & & \\
\hline & Log Likelihood & -133.731 & -411.697 & & & & \\
\hline & $N$ & 133 & 121 & & & & \\
\hline
\end{tabular}




\begin{tabular}{|c|c|c|c|c|c|c|c|}
\hline & & (1) & (2) & (3 a) & (3 b) & (3c) & (3d) \\
\hline \multirow{9}{*}{ 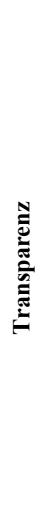 } & Twinning & $\begin{array}{l}.172 \\
(.141)\end{array}$ & $\begin{array}{l}.067 \\
(.118)\end{array}$ & & & & \\
\hline & Admin. Sozialisation & & $\begin{array}{l}.009 \\
(.080)\end{array}$ & & & & \\
\hline & Auslandsaufenthalt & & $\begin{array}{l}-.045 \\
(.082)\end{array}$ & & & & \\
\hline & Ausländ. Medien & & $\begin{array}{l}-.014 \\
(.095)\end{array}$ & & & & \\
\hline & Andere Programme & & $\begin{array}{l}.203 \\
(.081)^{*}\end{array}$ & & & & \\
\hline & $R^{2}$ & .010 & .058 & & & & \\
\hline & $A I C$ & 172.917 & 774.551 & & & & \\
\hline & Log Likelihood & -83.459 & -380.276 & & & & \\
\hline & $N$ & 132 & 121 & & & & \\
\hline \multirow{11}{*}{ 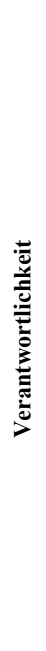 } & Twinning & $\begin{array}{l}.259 \\
(.203)\end{array}$ & $\begin{array}{l}.201 \\
(.183)\end{array}$ & $\begin{array}{l}-.038 \\
(.188)\end{array}$ & $\begin{array}{l}.664 \\
(.273)^{*}\end{array}$ & & \\
\hline & Admin. Sozialisation & & $\begin{array}{l}-.025 \\
(.115)\end{array}$ & $\begin{array}{l}-.038 \\
(.116)\end{array}$ & $\begin{array}{l}-.038 \\
(.116)\end{array}$ & & \\
\hline & Auslandsaufenthalt & & $\begin{array}{l}-.070 \\
(.118)\end{array}$ & $\begin{array}{l}-.120 \\
(.122)\end{array}$ & $\begin{array}{l}.120 \\
(.122)\end{array}$ & & \\
\hline & Ausländ. Medien & & $\begin{array}{l}.061 \\
(.153)\end{array}$ & $\begin{array}{l}.083 \\
(.150)\end{array}$ & $\begin{array}{l}.083 \\
(.150)\end{array}$ & & \\
\hline & Andere Programme & & $\begin{array}{l}.262 \\
(.117) *\end{array}$ & $\begin{array}{l}.268 \\
(.016)^{*}\end{array}$ & $\begin{array}{l}.268 \\
(.116) *\end{array}$ & & \\
\hline & $\begin{array}{l}\text { Twin. } \mathrm{x} 1 \text { : Auslandsauf- } \\
\text { enthalt }\end{array}$ & & & $\begin{array}{l}.702 \\
(.331)^{*}\end{array}$ & & & \\
\hline & $\begin{array}{l}\text { Twin. } \mathrm{x} \text { 1: kein Aus- } \\
\text { landsaufenthalt }\end{array}$ & & & & $\begin{array}{l}-.702 \\
(.331)^{*}\end{array}$ & & \\
\hline & $R^{2}$ & .011 & .051 & .070 & .070 & & \\
\hline & $A I C$ & 282.603 & 586.600 & 702.004 & 702.004 & & \\
\hline & Log Likelihood & -138.302 & -421.300 & -343.002 & -343.002 & & \\
\hline & $N$ & 126 & 117 & 117 & 117 & & \\
\hline
\end{tabular}

Multiple Regressionsanalysen (MLMV); Regressionskoeffizienten sind nicht standardisiert; Standardfehler in Klammern; Fälle mit fehlenden Werten listenweise ausgeschlossen;

t $p \leq 08, * p \leq .05, * * p \leq .01, * * * p \leq .001$. 
Tina Freyburg: Demokratisierung durch Zusammenarbeit?

IVc: Regressionsergebnisse - Das Twinning-Projekt »Gesundheitskontrolle»

\begin{tabular}{|c|c|c|c|c|c|}
\hline & & (1) & (2) & (3a) & (3b) \\
\hline \multirow{9}{*}{ 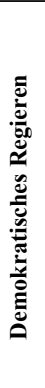 } & Twinning & $-.032(.071)$ & $.014(.081)$ & & \\
\hline & Admin. Sozialisation & & $-.047(.076)$ & & \\
\hline & Auslandsaufenthalt & & $-.051(.079)$ & & \\
\hline & Ausländ. Medien & & $-.062(.089)$ & & \\
\hline & Andere Programme & & $.222(.078) * *$ & & \\
\hline & $R^{2}$ & .001 & .089 & & \\
\hline & $A I C$ & 215.990 & 728.534 & & \\
\hline & Log Likelihood & -104.995 & -357.267 & & \\
\hline & $N$ & 110 & 103 & & \\
\hline \multirow{11}{*}{ 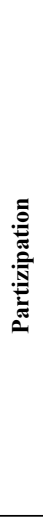 } & Twinning & $-.054(.101)$ & $.063(.122)$ & $-.169(.168)$ & $.266(.149)$ \\
\hline & Admin. Sozialisation & & $-.161(.110)$ & $-.139(.108)$ & $-.139(.108)$ \\
\hline & Auslandsaufenthalt & & $-.098(.111)$ & $-.185(.131)$ & $.185(.131)$ \\
\hline & Ausländ. Medien & & $-.143(.130)$ & $-.181(.132)$ & $-.181(.132)$ \\
\hline & Andere Programme & & $.246(.113)^{*}$ & $.251(.111)^{*}$ & $.251(.111)^{*}$ \\
\hline & $\begin{array}{l}\text { Twin. } \times 1 \text { : Auslandsauf- } \\
\text { enthalt }\end{array}$ & & & $.435(.217)^{*}$ & \\
\hline & $\begin{array}{l}\text { Twin. } \times 1 \text { : kein Aus- } \\
\text { landsaufenthalt }\end{array}$ & & & & $-.435(.217)^{*}$ \\
\hline & $R^{2}$ & .001 & .073 & .094 & .094 \\
\hline & $A I C$ & 357.259 & 959.985 & 900.858 & 900.858 \\
\hline & Log Likelihood & -175.630 & -472.992 & -442.429 & -442.429 \\
\hline & $N$ & 133 & 121 & 121 & 121 \\
\hline \multirow{11}{*}{ 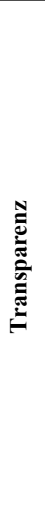 } & Twinning & $-.133(.090)$ & $-.155(.090)^{\dagger}$ & $.144(.131)$ & $-.208(.103)^{*}$ \\
\hline & Admin. Sozialisation & & $.020(.080)$ & $.011(.080)$ & $.011(.080)$ \\
\hline & Auslandsaufenthalt & & $-.028(.083)$ & $-.008(.086)$ & $-.008(.086)$ \\
\hline & Ausländ. Medien & & $-.002(.097)$ & $.045(.107)$ & $-.045(.107)$ \\
\hline & Andere Programme & & $.193(.080)^{* * *}$ & $.188(.080)^{* * *}$ & $.188(.080)^{* *}$ \\
\hline & $\begin{array}{l}\text { Twin. } \mathrm{x} \text { 1: ausländ. Me- } \\
\text { dien }\end{array}$ & & & $-.352(.174)^{*}$ & \\
\hline & $\begin{array}{l}\text { Twin. } \times 1 \text { : keine aus- } \\
\text { länd. Medien }\end{array}$ & & & & $.352(.174) *$ \\
\hline & $R^{2}$ & .013 & .076 & .089 & .089 \\
\hline & $A I C$ & 280.348 & 878.066 & 735.132 & 735.132 \\
\hline & Log Likelihood & -137.174 & -432.033 & -359.566 & -359.566 \\
\hline & $N$ & 132 & 121 & 121 & 121 \\
\hline
\end{tabular}




\begin{tabular}{|c|c|c|c|c|c|}
\hline & & (1) & (2) & (3a) & (3b) \\
\hline \multirow{9}{*}{ 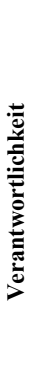 } & Twinning & $.075(.122)$ & $.127(.133)$ & & \\
\hline & Admin. Sozialisation & & $-.048(.118)$ & & \\
\hline & Auslandsaufenthalt & & $-.084(.122)$ & & \\
\hline & Ausländ. Medien & & $.055(.158)$ & & \\
\hline & Andere Programme & & $.262(.117)^{*}$ & & \\
\hline & $R^{2}$ & .002 & .050 & & \\
\hline & $A I C$ & 377.908 & 950.349 & & \\
\hline & Log Likelihood & -185.954 & -468.174 & & \\
\hline & $N$ & 126 & 117 & & \\
\hline
\end{tabular}

Multiple Regressionsanalysen (MLMV); Regressionskoeffizienten sind nicht standardisiert; Standardfehler in Klammern; Fälle mit fehlenden Werten listenweise ausgeschlossen; ${ }^{+} p \leq .06,{ }^{*} p \leq .05,{ }^{* *} p \leq 01$. 
Tina Freyburg: Demokratisierung durch Zusammenarbeit?

IVd: Regressionsergebnisse - Das Twinning-Projekt »Umwelt«

\begin{tabular}{|c|c|c|c|c|c|}
\hline & & (1) & (2) & (3a) & (3b) \\
\hline \multirow{11}{*}{ 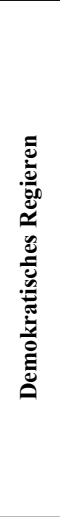 } & Twinning & $.179(.081)^{*}$ & $.163(.094)$ & $-.248(.117)^{*}$ & $.195(.096)^{*}$ \\
\hline & Admin. Sozialisation & & $-.015(.072)$ & $-.018(.072)$ & $-.018(.072)$ \\
\hline & Auslandsaufenthalt & & $-.039(076)$ & $-.025(.078)$ & $-.025(.078)$ \\
\hline & Ausländ. Medien & & $-.093(.092)$ & $-.120(.097)$ & $.120(.097)$ \\
\hline & Andere Programme & & $.208(.077)^{* * *}$ & $.217(.077)^{* *}$ & $.217(.077)^{* * *}$ \\
\hline & $\begin{array}{l}\text { Twin. x 1: ausländ. Me- } \\
\text { dien }\end{array}$ & & & $.442(.161)^{* *}$ & \\
\hline & $\begin{array}{l}\text { Twin. x 1: keine aus- } \\
\text { länd. Medien }\end{array}$ & & & & $-.442(.161)^{* *}$ \\
\hline & $R^{2}$ & .030 & .111 & .122 & .122 \\
\hline & $A I C$ & 195.533 & 695.634 & 493.798 & 493.798 \\
\hline & Log Likelihood & -94.767 & -340.634 & -238.899 & -238.899 \\
\hline & $N$ & 110 & 103 & 103 & 103 \\
\hline \multirow{9}{*}{ 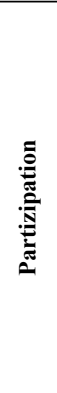 } & Twinning & $.438(.091)^{* * *}$ & $.401(.103)^{* * *}$ & & \\
\hline & Admin. Sozialisation & & $-.093(.102)$ & & \\
\hline & Auslandsaufenthalt & & $-.094(.104)$ & & \\
\hline & Ausländ. Medien & & $-.178(.127)$ & & \\
\hline & Andere Programme & & $.183(.107)$ & & \\
\hline & $R^{2}$ & .092 & .140 & & \\
\hline & AIC & 360.973 & 948.425 & & \\
\hline & Log Likelihood & -177.487 & -467.213 & & \\
\hline & $N$ & 133 & 121 & & \\
\hline \multirow{11}{*}{ 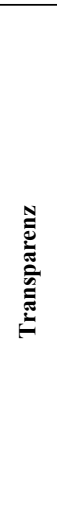 } & Twinning & $.035(.101)$ & $.121(.084)$ & $-.043(.081)$ & $.393(.148)^{* * *}$ \\
\hline & Admin. Sozialisation & & $.026(.081)$ & $.012(.079)$ & $.012(.079)$ \\
\hline & Auslandsaufenthalt & & $-.040(.082)$ & $-.106(.089)$ & $.106(.089)$ \\
\hline & Ausländ. Medien & & $-.029(.095)$ & $.012(.096)$ & $.012(.096)$ \\
\hline & Andere Programme & & $.186(.080)^{*}$ & $.174(.079)^{*}$ & $.174(.079)^{*}$ \\
\hline & $\begin{array}{l}\text { Twin. } \mathrm{x} \text { 1: Auslandsauf- } \\
\text { enthalt }\end{array}$ & & & $.436(.169)^{* *}$ & \\
\hline & $\begin{array}{l}\text { Twin. x 1: kein Aus- } \\
\text { landsaufenthalt }\end{array}$ & & & & $-.436(.169)^{* *}$ \\
\hline & $R^{2}$ & .001 & .067 & .100 & 100 \\
\hline & $A I C$ & 286.390 & 860.657 & 778.467 & 778.467 \\
\hline & Log Likelihood & -140.195 & -423.329 & -381.233 & -381.233 \\
\hline & $N$ & 132 & 121 & 121 & 121 \\
\hline
\end{tabular}




\begin{tabular}{|c|c|c|c|c|c|}
\hline & & (1) & (2) & (3 a) & (3 b) \\
\hline \multirow{9}{*}{ 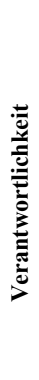 } & Twinning & $-.104(.150)$ & $-.205(.170)$ & & \\
\hline & Admin. Sozialisation & & $-.069(.122)$ & & \\
\hline & Auslandsaufenthalt & & $-.071(.119)$ & & \\
\hline & Ausländ. Medien & & $.100(.154)$ & & \\
\hline & Andere Programme & & $.277(.123)^{*}$ & & \\
\hline & $R^{2}$ & .004 & .057 & & \\
\hline & $A I C$ & 369.190 & 922.311 & & \\
\hline & Log Likelihood & -181.595 & -454.156 & & \\
\hline & $N$ & 126 & 117 & & \\
\hline
\end{tabular}

Multiple Regressionsanalysen (MLMV); Regressionskoeffizienten sind nicht standardisiert; Standardfehler in Klammern; Fälle mit fehlenden Werten listenweise ausgeschlossen; * $\leq \leq 05, *^{*} p \leq 01, *_{*}^{*} p \leq 001$. 